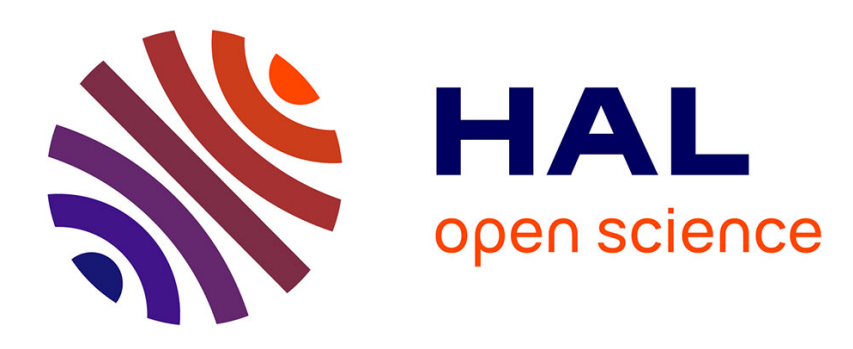

\title{
A mortar spectral element method for 3D Maxwell's equations
}

\author{
Tahar Z Boulmezaoud, Mohammed El Rhabi
}

\section{To cite this version:}

Tahar Z Boulmezaoud, Mohammed El Rhabi. A mortar spectral element method for 3D Maxwell's equations. IMA Journal of Numerical Analysis, 2005, 25 (3), pp.577 - 610. 10.1093/imanum/dri010 . hal-01813484

\section{HAL Id: hal-01813484 \\ https://hal-enpc.archives-ouvertes.fr/hal-01813484}

Submitted on 12 Jun 2018

HAL is a multi-disciplinary open access archive for the deposit and dissemination of scientific research documents, whether they are published or not. The documents may come from teaching and research institutions in France or abroad, or from public or private research centers.
L'archive ouverte pluridisciplinaire HAL, est destinée au dépôt et à la diffusion de documents scientifiques de niveau recherche, publiés ou non, émanant des établissements d'enseignement et de recherche français ou étrangers, des laboratoires publics ou privés. 
archives-ouvertes

\section{A mortar spectral element method for 3D Maxwell's equations}

Tahar Boulmezaoud, Mohammed El Rhabi

\section{To cite this version:}

Tahar Boulmezaoud, Mohammed El Rhabi. A mortar spectral element method for 3D Maxwell's equations. IMA Journal of Numerical Analysis, Oxford University Press (OUP), 2005, 25 (3), pp.577

- 610. <10.1093/imanum/dri010>. <hal-01812923>

\section{HAL Id: hal-01812923 \\ https://hal-enpc.archives-ouvertes.fr/hal-01812923}

Submitted on 12 Jun 2018

HAL is a multi-disciplinary open access archive for the deposit and dissemination of scientific research documents, whether they are published or not. The documents may come from teaching and research institutions in France or abroad, or from public or private research centers.
L'archive ouverte pluridisciplinaire HAL, est destinée au dépôt et à la diffusion de documents scientifiques de niveau recherche, publiés ou non, émanant des établissements d'enseignement et de recherche français ou étrangers, des laboratoires publics ou privés. 


\title{
A mortar spectral element method for 3D Maxwell's equations
}

\author{
TAHAR Z. BOULMEZAOUD† \\ Laboratoire de Mathématiques Appliquées, \\ Université de Pau et des pays de l'Adour, \\ Avenue de l'Univeristé, BP 1155, 64013 Pau Cedex, France \\ AND \\ Mohammed El RHABI† \\ Laboratoire Jacques-Louis Lions, Université Paris VI, \\ 175, rue du Chevaleret, BP 187, 75013 Paris, France \\ Prepint submitted to IMA Journal of Numerical Analysis (2005)
}

\begin{abstract}
In this paper we propose a mortar spectral element method for solving Maxwell's equations in 3D bounded cavities. The method is based on a non-conforming decomposition of the domain into the union of non-overlapping parallelepipeds. After proving an error estimate, we present some 3D computational results which confirm the performance of the method.
\end{abstract}

Keywords: Maxwell's equations; mortar method; spectral elements, Galerkin approximations.

\section{Introduction}

The last two decades have seen a rapid development of numerical methods for solving Maxwell's equations in 2D and in 3D bounded regions of space. Let us recall e.g. the works of Hoppe $(1981,1982)$, Monk (1992a,b, 1993, 2003), Beck et al. (1999), Beck \& Hiptmair (1999), Hiptmair (1999), Costabel (1991), Costabel \& Dauge (1999, 2000), Buffa et al. (2003), Toselli (2000) and Toselli et al. (2001). Among the existing methods, domain decomposition techniques turned out to be very efficient since they bring a serious flexibility in the discretization of the original problem.

Our goal in this paper is to use a mortar spectral element method for approximating time-dependent and time-harmonic Maxwell's equations in a 3D bounded cavity.

The mortar method was introduced in Bernardi et al. (1990a,b) for coupling spectral and finite element approximations. It has mainly two advantages. Firstly, it allows the coupling of different, independent and non-matching numerical discretizations inside the domain of interest. Secondly, it is naturally predisposed to parallel computation (see, e.g. Fisher \& Patera, 1991).

Mortar method with finite elements, spectral elements or wavelets were successfully applied to second-order elliptic problems (see, e.g. Bernardi et al., 1990b; Wohlmuth, 2000; Bertoluzza \& Perrier, 2001). It was also applied to several other problems including fourth-order equations (see Belhachmi \& Bernardi, 1994), Stokes equation (Débit \& Maday, 1987) and 2D and 3D Maxwell's equations (with finite elements) (see Rapetti, 2000; Ben Belgacem et al., 2001).

Let us describe the Maxwell's equations we deal with in this paper. Let $\Omega$ be a bounded open set of $\mathbb{R}^{3}$, with Lipschitz boundary $\partial \Omega$ and a unit outward normal $\boldsymbol{n}$. Assume also that $\Omega$ is occupied by a 
heterogeneous, linear and isotropic material and that its boundary $\partial \Omega$ is a perfect conductor. If $\boldsymbol{E}(\boldsymbol{x}, t)$ and $\boldsymbol{H}(\boldsymbol{x}, t)$ denote, respectively, the electric and magnetic fields in $\Omega$, then Maxwell's equations state that

$$
\begin{aligned}
\mu \partial_{t} \boldsymbol{H}+\operatorname{curl} \boldsymbol{E} & =\mathbf{0}, \\
\epsilon \partial_{t} \boldsymbol{E}-\operatorname{curl} \boldsymbol{H} & =-\boldsymbol{j}, \\
\operatorname{div}(\mu \boldsymbol{H}) & =0, \\
\operatorname{div}(\epsilon \boldsymbol{E}) & =\rho,
\end{aligned}
$$

with

$$
\frac{\partial \rho}{\partial t}+\operatorname{div} \boldsymbol{j}=0
$$

The boundary conditions we consider here are of the form

$$
\boldsymbol{E} \times \boldsymbol{n}=\mathbf{0} \quad \text { on } \partial \Omega, \quad \mu \boldsymbol{H} \cdot \boldsymbol{n}=0 \quad \text { on } \partial \Omega .
$$

Here and below $\rho$ and $\boldsymbol{j}$ denote, respectively, the charge and the electric current densities. The coefficient $\mu(\boldsymbol{x})$ is the magnetic permeability of the material in $\Omega$ and $\epsilon(\boldsymbol{x})$ is its electric permittivity. We suppose that $\epsilon(\boldsymbol{x})$ and $\mu(\boldsymbol{x})$ are real, bounded and positive functions depending on the space variable $\boldsymbol{x}$, i.e. there exist four constants $\epsilon_{0}, \epsilon_{\infty}, \mu_{0}$ and $\mu_{\infty}$ such that

$$
0<\epsilon_{0} \leqslant \epsilon(\boldsymbol{x}) \leqslant \epsilon_{\infty} \quad \text { and } \quad 0<\mu_{0} \leqslant \mu(\boldsymbol{x}) \leqslant \mu_{\infty} \quad \text { a.e. in } \Omega .
$$

In a time-dependent regime, the evolution equations (1.1) are completed with the initial conditions

$$
\boldsymbol{E}(\boldsymbol{x}, t=0)=\boldsymbol{E}_{0}(\boldsymbol{x}), \quad \boldsymbol{H}(\boldsymbol{x}, t=0)=\boldsymbol{H}_{0}(\boldsymbol{x}) .
$$

It is usual to rewrite system (1.1) in terms of one unknown, say the magnetic field $\boldsymbol{H}$ or the electric field $\boldsymbol{E}$. Here, we choose the formulation in terms of $\boldsymbol{H}$. Eliminating $\boldsymbol{E}$ from the equations above gives

$$
\mu \partial_{t}^{2} \boldsymbol{H}+\operatorname{curl}\left(\epsilon^{-1} \operatorname{curl} \boldsymbol{H}\right)=\operatorname{curl}\left(\epsilon^{-1} \boldsymbol{j}\right), \quad \operatorname{div}(\mu \boldsymbol{H})=0 .
$$

The magnetic field is subject to the boundary conditions

$$
\begin{aligned}
\mu \boldsymbol{H} \cdot \boldsymbol{n} & =0 & & \text { on } \partial \Omega, \\
\epsilon^{-1}(\operatorname{curl} \boldsymbol{H}-\boldsymbol{j}) \times \boldsymbol{n} & =\mathbf{0} & & \text { on } \partial \Omega,
\end{aligned}
$$

and to initial conditions (1.5). In a time-harmonic regime the magnetic field satisfies

$$
\operatorname{curl}\left(\epsilon^{-1} \operatorname{curl} \boldsymbol{H}\right)-\omega^{2} \mu \boldsymbol{H}=\operatorname{curl}\left(\epsilon^{-1} \boldsymbol{j}\right) .
$$

The space discretizations of (1.6) and (1.8) are often distinguished in the literature. Indeed, an implicit time-stepping of (1.6) leads usually to a system of the form (see, e.g. Hoppe, 1982)

$$
\begin{aligned}
\operatorname{curl}\left(\epsilon^{-1} \operatorname{curl} \boldsymbol{u}\right)+\tau \mu \boldsymbol{u} & =\operatorname{curl}\left(\epsilon^{-1} \boldsymbol{j}\right)+\boldsymbol{u}_{0} & & \text { in } \Omega, \\
\mu \boldsymbol{u} \cdot \boldsymbol{n} & =0 & & \text { on } \partial \Omega, \\
\operatorname{curl} \boldsymbol{u} \times \boldsymbol{n} & =\boldsymbol{j} \times \boldsymbol{n} & & \text { on } \partial \Omega,
\end{aligned}
$$

where $\tau$ is a positive parameter. The weak formulation of this problem rests on the bilinear form $\left(\epsilon^{-1} \operatorname{curl} u, \operatorname{curl} v\right)+(\tau \mu \boldsymbol{u}, v)$ which is coercive on $H(\operatorname{curl} ; \Omega)$. 
In contrast, the time-harmonic equation (1.8), written in a weak form, involves the bilinear form $\left(\epsilon^{-1}\right.$ $\operatorname{curl} \boldsymbol{u}, \operatorname{curl} v)-\omega^{2}(\mu \boldsymbol{u}, \boldsymbol{v})$ which is not coercive either on $H(\operatorname{curl} ; \Omega)$ or on $H_{0}(\operatorname{div} ; \mu, \Omega) \cap H(\operatorname{curl} ; \Omega)$ even if $\omega>0$. Two techniques might be used to deal with this problem. One of these is to penalize the weak problem by adding the term $(\operatorname{div}(\mu \boldsymbol{u}), \operatorname{div}(\mu \boldsymbol{v}))$ (actually, this term is 0 when $\boldsymbol{u}=\boldsymbol{H}$ ) (see, e.g. Hoppe, 1981, or Costabel, 1991). In this case, the bilinear form

$$
\left(\epsilon^{-1} \operatorname{curl} u, \operatorname{curl} v\right)+(\operatorname{div}(\mu \boldsymbol{u}), \operatorname{div}(\mu v))
$$

is coercive on the space $H_{0}(\operatorname{div} \boldsymbol{u}, \mu, \Omega) \cap H(\operatorname{curl} ; \Omega)$. Unfortunately, as will be noted in Remark 1 below, this penalty method employed with mortar spectral elements induces some complications which are still unresolved. The second technique, which we expose in Section 2.2, consists of finding the solution of (1.8) as the limit of a quickly converging sequence of solutions of positive definite problems of form (1.9). A considerable advantage of such a technique is the possibility of using in practice a common method for solving time-dependent and time-harmonic Maxwell's equations. Thus, we choose to use the second technique in this paper.

Let us notice that if $\Omega$ is a non-convex polyhedron, then the magnetic field can have some singularities which are due to reentrant corners and edges. The reader can refer to Costabel \& Dauge (2000) and references therein for a characterization of these singularities. In that case, it is well known that $H^{1}$ elements are not sufficient to approximate the singular part of the solution (see for instance Costabel, 1991, or Costabel \& Dauge, 2000). Similarly, singularities could appear also at interfaces if the parameters $\epsilon$ and $\mu$ are discontinuous (see, e.g. Costabel \& Dauge, 1999). The analysis and the approximation of these singularities are beyond the scope of this paper. Here, we shall suppose that the solution we look for is piecewise smooth (see the subsequent Theorem 1), and we do not make for the moment any other assumption, either on the domain $\Omega$ or on the coefficients $\epsilon$ and $\mu$. Of course, this smoothness assumption is plausible if $\Omega$ is convex or if it has a regular boundary and if the coefficients $\epsilon$ and $\mu$ are constant or globally smooth (see, e.g. Lemma 2.3 in Monk, 1992a). In other situations, the method we expose here could be used only for computing the smooth part of the solution. The singular part must be treated separately.

An outline of this paper is as follows. In Section 2, we recall and state briefly some definitions and properties of the function spaces used as a framework. Then, we prove that each solution of the time-harmonic Maxwell's equations can be obtained as the limit of a rapidly converging sequence of solutions of a coercive problem.

Section 3 is devoted to the presentation of a mortar method with spectral elements for Maxwell's equations and to the proof of its numerical convergence.

The purpose of Section 4 is twofold. Firstly, we show that in practice the implementation of the mortar spectral element could be simplified by means of a theoretical treatment of the transmission conditions. Secondly, we display some 3D computational results which confirm the convergence of the method in several cases.

\section{Preliminaries}

\subsection{Notations}

In the sequel, for any integer $d \geqslant 1$, any open set $\mathcal{O}$ of $\mathbb{R}^{d}(d=1,2,3)$ and any positive integers $N_{1}, \ldots, N_{d}, \mathbb{P}_{N_{1}, \ldots, N_{d}}(\mathcal{O})$, stands for the space of all the polynomial functions on $\mathcal{O}$ whose degree with respect to the variable $x_{i}, 1 \leqslant i \leqslant d$, is less than or equal to $N_{i}$. If $N_{1}=\cdots=N_{d}=N$, then we write $\mathbb{P}_{N}(\mathcal{O})$ instead of $\mathbb{P}_{N_{1}, \ldots, N_{d}}(\mathcal{O})$. As usual, $L^{2}(\mathcal{O})$ refers to the space of square integrable functions 
on $\mathcal{O}$. This space is equipped with the inner product

$$
(u, v)_{\mathcal{O}}=\int_{\mathcal{O}} u(\boldsymbol{x}) v(\boldsymbol{x}) \mathrm{d} \boldsymbol{x},
$$

and with the associated norm $\|u\|_{0, \mathcal{O}}=(u, u)_{\mathcal{O}}^{1 / 2}$. The subscript $\mathcal{O}$ will be dropped in these notations when the domain is $\Omega$.

In what follows $H^{s}(\mathcal{O}), s$ being real, will be the usual Sobolev space of order $s$ and $\|\cdot\|_{s, \mathcal{O}}$ its norm. When $\mathcal{O}$ is an open set of $\mathbb{R}^{3}$ we write

$$
H(\operatorname{curl} ; \mathcal{O})=\left\{v \in L^{2}(\mathcal{O})^{3}, \operatorname{curl} v \in L^{2}(\mathcal{O})^{3}\right\} .
$$

This is a Hilbert space equipped with the inner product

$$
(u, v)_{\operatorname{curl}, \mathcal{O}}=(\operatorname{curl} u, \operatorname{curl} v)_{0, \mathcal{O}}+(u, v)_{0, \mathcal{O}},
$$

and with the norm

$$
\|\boldsymbol{u}\|_{H(\operatorname{curl} ; \mathcal{O})}=\left(\|\boldsymbol{v}\|_{0, \mathcal{O}}^{2}+\|\operatorname{curl} \boldsymbol{v}\|_{0, \mathcal{O}}^{2}\right)^{1 / 2} .
$$

Recall that if $\mathcal{O}$ is bounded and has a Lipschitzian boundary, then the tangential trace operator $\boldsymbol{u} \mapsto \boldsymbol{u} \times \boldsymbol{n}$ is well defined and is continuous from $H(\mathbf{c u r l} ; \mathcal{O})$ into $H^{-1 / 2}(\partial \mathcal{O})^{3}$. Moreover, the following Green's formula holds

$$
\forall \boldsymbol{v} \in H(\operatorname{curl} ; \mathcal{O}), \quad \forall \boldsymbol{w} \in H^{1}(\mathcal{O})^{3}, \quad(\operatorname{curl} \boldsymbol{v}, \boldsymbol{w})=(\boldsymbol{v}, \operatorname{curl} \boldsymbol{w})+\langle\boldsymbol{v} \times \boldsymbol{n}, \boldsymbol{w}\rangle_{\partial \mathcal{O}} .
$$

Let $H_{0}(\mathbf{c u r l} ; \mathcal{O})$ refer to the subspace of vector fields in $H(\mathbf{c u r l} ; \mathcal{O})$ with a vanishing tangential component on $\partial \mathcal{O}$.

Finally, we introduce the space

$$
H(\operatorname{div} ; \mu, \Omega)=\left\{\boldsymbol{v} \in L^{2}(\Omega)^{3}, \operatorname{div}(\mu \boldsymbol{v}) \in L^{2}(\Omega)\right\},
$$

equipped with the norm

$$
\|\boldsymbol{u}\|_{H(\operatorname{div} ; \mu, \Omega)}=\left(\|\boldsymbol{v}\|_{0, \Omega}^{2}+\|\operatorname{div}(\mu \boldsymbol{v})\|_{0, \Omega}^{2}\right)^{1 / 2},
$$

and its subspace

$$
X_{T}(\mu ; \Omega)=\{\boldsymbol{u} \in H(\operatorname{curl} ; \Omega) \cap H(\operatorname{div} ; \mu, \Omega) \mid \mu \boldsymbol{u} \cdot \boldsymbol{n}=0 \text { on } \partial \Omega\},
$$

endowed with the norm

$$
\|\boldsymbol{u}\|_{X_{T}(\mu ; \Omega)}=\left\{\|\boldsymbol{u}\|_{0, \Omega}^{2}+\|\operatorname{curl} \boldsymbol{u}\|_{0, \Omega}^{2}+\|\operatorname{div}(\mu \boldsymbol{u})\|_{0, \Omega}^{2}\right\}^{1 / 2} .
$$

The following semi-norm on $X_{T}(\mu ; \Omega)$ will be useful subsequently

$$
|\boldsymbol{u}|_{X_{T}(\mu ; \Omega)}=\left\{\|\operatorname{curl} \boldsymbol{u}\|_{0, \Omega}^{2}+\|\operatorname{div}(\mu \boldsymbol{u})\|_{0, \Omega}^{2}\right\}^{1 / 2} .
$$

Actually, this semi-norm is a norm on $X_{T}(\mu ; \Omega)$ equivalent to the norm (2.2) (see Monk, 2003, and Costabel, 1991, see also El Rhabi, 2002, for a detailed proof).

Consequently, we set

$$
\omega_{0}=\inf _{\boldsymbol{v} \in X_{T}(\mu ; \Omega) ; \operatorname{div}(\mu \boldsymbol{v})=0 ; \boldsymbol{v} \neq \mathbf{0}} \frac{\left\|\epsilon^{-1 / 2} \operatorname{curl} \boldsymbol{v}\right\|_{0, \Omega}}{\left\|\mu^{1 / 2} \boldsymbol{v}\right\|_{0, \Omega}} .
$$

This constant is strictly positive and depends only on $\Omega$ and on the parameters $\epsilon$ and $\mu$. 


\subsection{A relaxation method for time-harmonic Maxwell's equations}

As mentioned in the introduction, the solution of the time-harmonic problem (2.5) can be obtained as the limit of a sequence of solutions of problems of the form (1.9). The main goal of such a procedure is to prove that the numerical solution of both time-dependent and time-harmonic Maxwell's systems can be obtained in practice by solving a positive definite elliptic problem. In this preliminary section, we construct such a sequence and we prove its convergence.

Let us consider the time-harmonic Maxwell's system

$$
\begin{aligned}
\operatorname{curl}\left(\epsilon^{-1} \operatorname{curl} \boldsymbol{u}\right)-\omega^{2} \mu \boldsymbol{u} & =\operatorname{curl}\left(\epsilon^{-1} \boldsymbol{j}\right) & & \text { in } \Omega, \\
\operatorname{div}(\mu \boldsymbol{u}) & =0 & & \text { in } \Omega, \\
\epsilon^{-1}(\operatorname{curl} \boldsymbol{u}-\boldsymbol{j}) \times \boldsymbol{n} & =\mathbf{0} & & \text { on } \partial \Omega .
\end{aligned}
$$

It is well known that this problem satisfies the Fredholm alternative (see Leis, 1986, see also Dautray \& Lions, 1988, and Boulmezaoud \& El Rhabi, 2003).

Now, let us consider the following iteration procedure; given $\boldsymbol{u}_{0}=\mathbf{0}$, for any $k \geqslant 0$ find $\boldsymbol{u}_{k+1}$ as the solution of the weak problem

$$
\forall v \in X_{T}(\mu ; \Omega)\left(\epsilon^{-1} \operatorname{curl} \boldsymbol{u}_{k+1}, \operatorname{curl} v\right)+\tau\left(\mu \boldsymbol{u}_{k+1}, v\right)=\left(\omega^{2}+\tau\right)\left(\boldsymbol{u}_{k}, \mu v\right)+\left(\epsilon^{-1} \boldsymbol{j}, \operatorname{curl} v\right),
$$

where $\tau$ is a fixed real parameter not depending on $k$ and which will be chosen to ensure convergence. Observe that the bilinear form on the left-hand side of (2.6) is coercive if $\tau>-\omega_{0}^{2}$. It follows from the Lax-Milgram theorem that the sequence $\left(\boldsymbol{u}_{k}\right)_{k \geqslant 0}$ is well defined. The convergence of that sequence is stated as follows.

Proposition 1 Suppose that

$$
\omega<\omega_{0} \text { and } \tau>-\frac{\omega^{2}+\omega_{0}^{2}}{2} .
$$

Then, the sequence $\left(\boldsymbol{u}_{k}\right)_{k \geqslant 0}$ converges strongly in $X_{T}(\mu ; \Omega)$ to the unique solution of $(2.5)$ in $X_{T}(\mu ; \Omega)$. Furthermore, one has

$$
\left\|\boldsymbol{u}_{k}-\boldsymbol{u}\right\|_{X_{T}(\mu ; \Omega)} \leqslant \epsilon_{\infty}^{\frac{1}{2}}\left(\omega_{0}^{2}+|\tau|\right)^{\frac{1}{2}} \kappa^{k+1}\|\boldsymbol{u}\|_{Y_{T}(\Omega, \mu, \epsilon)}
$$

where

$$
\kappa=\frac{\left|\omega^{2}+\tau\right|}{\omega_{0}^{2}+\tau}<1 .
$$

Proof. Consider the sequence $\Phi_{k}=\boldsymbol{u}_{k}-\boldsymbol{u}$, where $\boldsymbol{u} \in X_{T}(\mu ; \Omega)$ is the solution of (2.5). Then, $\operatorname{div}\left(\mu \Phi_{k}\right)=0$ and

$$
\left(\epsilon^{-1} \operatorname{curl} \Phi_{k+1}, \operatorname{curl} v\right)+\tau\left(\Phi_{k+1}, \mu v\right)=\left(\omega^{2}+\tau\right)\left(\Phi_{k}, \mu v\right),
$$

for all $v \in X_{T}(\mu ; \Omega)$. It follows that

$$
\left(\omega_{0}^{2}+\tau\right)\left\|\sqrt{\mu} \Phi_{k+1}\right\|_{0, \Omega} \leqslant\left|\omega^{2}+\tau\right|\left\|\sqrt{\mu} \Phi_{k}\right\|_{0, \Omega} .
$$

Thus, if $\tau>-\omega_{0}^{2}$ one gets

$$
\left\|\sqrt{\mu} \Phi_{k+1}\right\|_{0, \Omega} \leqslant \kappa\left\|\sqrt{\mu} \Phi_{k}\right\|_{0, \Omega} .
$$


Hence,

$$
\left\|\sqrt{\mu} \Phi_{k+1}\right\|_{0, \Omega} \leqslant \kappa^{k+1}\|\sqrt{\mu} \boldsymbol{u}\|_{0, \Omega} .
$$

Moreover, from (2.8) then

$$
\begin{aligned}
\epsilon_{\infty}^{-1}\left|\Phi_{k+1}\right|_{X_{T}(\mu ; \Omega)}^{2} & \leqslant\left(-\inf (\tau, 0)\left\|\sqrt{\mu} \Phi_{k+1}\right\|_{0, \Omega}+\left|\omega^{2}+\tau\right|\left\|\sqrt{\mu} \Phi_{n}\right\|_{0, \Omega}\right)\left\|\sqrt{\mu} \Phi_{k+1}\right\|_{0, \Omega} \\
& \leqslant\left[-\inf (\tau, 0) \kappa^{k+1}+\left|\omega^{2}+\tau\right| \kappa^{k}\right] \kappa^{k+1}\|\sqrt{\mu} \boldsymbol{u}\|_{0, \Omega}^{2} \\
& \leqslant\left(\omega_{0}^{2}+|\tau|\right) \kappa^{2 k+2}\|\sqrt{\mu} \boldsymbol{u}\|_{0, \Omega}^{2} .
\end{aligned}
$$

If $\omega<\omega_{0}$ and $2 \tau>-\left(\omega_{0}^{2}+\omega^{2}\right)$, then $\kappa<1$. In that case the sequence $\left\{\boldsymbol{u}_{k}\right\}$ converges strongly to $\boldsymbol{u}$ in $X_{T}(\mu ; \Omega)$.

Of course, the definition of $\kappa$ in Proposition 1 suggests that the best choice for $\tau$ would be $\tau=$ $-\omega^{2}<0$. With this choice, one is back to the original problem and the relaxation procedure is useless. However, in order to get a positive definite bilinear form in the weak formulation, it is better to choose $\tau>0$ in which case one is led at each iteration to solving a coercive problem.

In the remainder of this paper we deal only with problem (1.9).

\section{The mortar method}

\subsection{The discrete space}

In what follows, for each integer $n \geqslant 0, L_{n}(x)$ stands for the Legendre polynomial of one variable and of degree equal to $n$. Recall that Legendre polynomials form an orthogonal system in $L^{2}(]-1,1[)$ and satisfy the following basic properties:

$$
L_{n}(1)=1, \quad \int_{-1}^{1} L_{n}(x)^{2} \mathrm{~d} x=\frac{2}{2 n+1}, \quad \int_{-1}^{1} L_{n}^{\prime}(x)^{2} \mathrm{~d} x=n(n+1),
$$

for each integer $n \geqslant 0$.

Given an integer $N \geqslant 2$, the Gauss-Lobatto grid on ] - 1, 1[ of order $N$ is formed by the zeros $\xi_{N, 0}=-1<\xi_{N, 1}<\cdots<\xi_{N, N}=1$ of the polynomial $\left(1-x^{2}\right) L_{N}^{\prime}(x)$ (see, e.g. Davis \& Rabinowitz, 1985; Szegö, 1978; Bernardi \& Maday, 1992). Moreover, there exist positive weights $\rho_{N, 0}, \ldots, \rho_{N, N}$ such that

$$
\forall p \in \mathbb{P}_{2 N-1}(]-1,1[), \quad \int_{-1}^{1} p(x) \mathrm{d} x=\sum_{i=0}^{N} \rho_{N, i} p\left(\xi_{N, i}\right) .
$$

Suppose now that $\Omega$ has a non-overlapping partition of the form

$$
\bar{\Omega}=\bigcup_{k=1}^{K} \bar{\Omega}_{k}
$$

where $\Omega_{1}, \ldots, \Omega_{K}$ are $K$ disjoint axiparallel rectangular parallelepipeds of $\mathbb{R}^{3}$. Assume that the intersection of two sub-domains is either empty or a point or a segment or a rectangle.

The skeleton $\mathcal{S}$ of this decomposition is defined by

$$
\mathcal{S}=\overline{\left(\bigcup_{k=1}^{K} \bigcup_{j=1}^{K} \partial \Omega_{k} \cap \partial \Omega_{j}\right)} .
$$



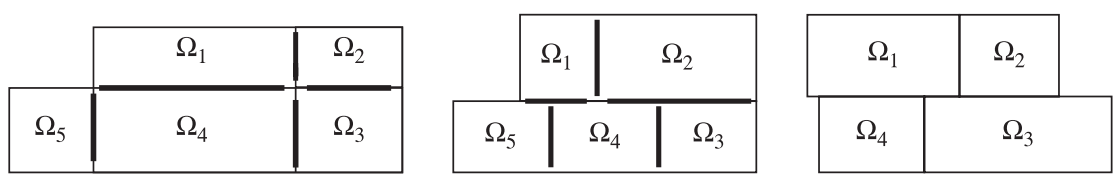

FIG. 1. A 2D example of a conforming decomposition (left). A 2D illustration of a non-conforming decomposition (centre). A 2D illustration of an excluded domain decomposition since some mortars cannot be chosen as the whole edges of sub-domains (right).

Suppose that this skeleton admits a partition of the form

$$
\mathcal{S}=\overline{\bigcap_{s=1}^{m} F_{s}^{-}},
$$

where, for each $s \leqslant m, F_{s}^{-}$is a whole face of $\Omega_{k(s)}$ for some $k(s) \leqslant K$. Let us underline that this last assumption is not restrictive since it allows conforming and non-conforming geometrical decompositions (see 2D illustrations in Fig. 1). However, it excludes some domain decompositions for which some of the faces $F_{s}^{-}$could not be chosen as complete faces of sub-domains (see the 2D illustration in Fig. 1).

The faces $F_{s}^{-}, s=1, \ldots, m$, are called mortar faces.

In the sequel, given an integer $s \leqslant m$, we write

$$
\begin{aligned}
& \mathbb{E}(s)=\left\{\ell \leqslant K \mid \partial \Omega_{\ell} \cap F_{s}^{-} \neq \emptyset\right\}, \\
& \widetilde{\mathbb{E}}(s)=\mathbb{E}(s)-\{k(s)\} .
\end{aligned}
$$

We call the slave side of $F_{s}^{-}$the face $F_{s}^{-}$itself considered as a part of the boundary of $\Omega_{k(s)}$. By its master side we mean $F_{s}^{-}$considered as a part of the boundary of $\bigcup_{\ell \in \widetilde{\mathbb{E}}(s)} \Omega_{\ell}$. If $u$ is a function on $\Omega$, then $u^{+}$refers to its value on the master side of the skeleton, while $u^{-}$refers to its value on the slave side. The jump of $u$ across the skeleton is given by $[u]=u^{+}-u^{-}$. Consequently, the restriction of any vector field in $H(\mathbf{c u r l} ; \Omega)$ to a sub-domain $\Omega_{k}$ is in $H\left(\right.$ curl; $\left.\Omega_{k}\right)$ and satisfies the transmission condition

$$
\left[\boldsymbol{u} \times \boldsymbol{n}_{k}\right]=0
$$

at each interface $\partial \Omega_{k} \cap \partial \Omega_{\ell}$. Notice that for almost every $\boldsymbol{x} \in F_{s}^{-}$there exists a unique $\ell \in \widetilde{\mathbb{E}}(s)$ such that $x \in \partial \Omega_{k(s)} \cap \partial \Omega_{\ell}$.

Now, consider $K$ integers $N_{1}, \ldots, N_{K}$ such that $N_{k} \geqslant 2$ for all $k \leqslant K$ and define the global discretization parameter

$$
\delta=\left(N_{1}, \ldots, N_{K}\right) \in \mathbb{N}^{K} .
$$

For any positive integer $j \leqslant N_{\min }, \delta-j$ will be the multi-index $\left(N_{1}-j, \ldots, N_{K}-j\right)$. We set

$$
\begin{aligned}
N_{\max } & =\max _{1 \leqslant k \leqslant K} N_{k}, \\
N_{\min } & =\min _{1 \leqslant k \leqslant K} N_{k}, \\
M_{s} & =N_{k(s)}, \\
\eta_{\delta} & =\max _{1 \leqslant s \leqslant m} \frac{\max _{\ell \in \mathbb{E}(s)} N_{\ell}}{\min _{\ell \in \mathbb{E}(s)} N_{\ell}} \leqslant \frac{N_{\max }}{N_{\min }} .
\end{aligned}
$$




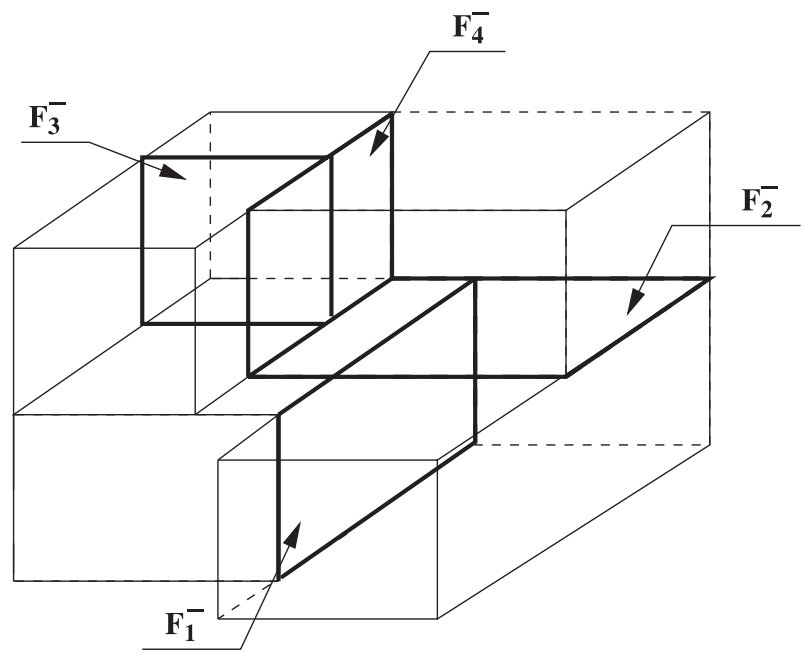

FIG. 2. An example of the mortar decomposition in a 3D configuration.

Observe that

$$
\forall s \leqslant m, \quad \forall \ell \in \mathbb{E}(s), \quad \eta_{\delta}^{-1} N_{\ell} \leqslant M_{s} \leqslant \eta_{\delta} N_{\ell}
$$

Now, to each sub-domain $\Omega_{k}, k=1, \ldots, K$, we associate a Gauss-Lobatto grid

$$
\Xi^{k}=\left\{\boldsymbol{\xi}_{\alpha}^{k}=\left(\xi_{N_{k}, i}^{k}, \xi_{N_{k}, j}^{k}, \xi_{N_{k}, \ell}^{k}\right) ; \alpha=(i, j, \ell) \text { with }|\alpha|=\sup (i, j, \ell) \leqslant N_{k}\right\},
$$

obtained by translation and homothety in each direction of the nodes $\xi_{N_{k}, 0}, \xi_{N_{k}, 1}, \ldots, \xi_{N_{k}, N_{k}}$.

The corresponding weights $\rho_{\alpha}^{(k)},|\alpha| \leqslant N_{k}$, are given by

$$
\rho_{\alpha}^{(k)}=\rho_{N_{k}, i} \rho_{N_{k}, j} \rho_{N_{k}, \ell} \frac{\left|\Omega_{k}\right|}{8}, \quad \text { with } \alpha=(i, j, \ell) .
$$

Define also the discrete product on the space of continuous functions on $\bar{\Omega}_{k}$ by

$$
(u, v)_{\Omega_{k}, N_{k}}=\sum_{|\alpha| \leqslant N_{k}} \rho_{\alpha}^{(k)} u\left(\boldsymbol{\xi}_{\alpha}^{k}\right) v\left(\boldsymbol{\xi}_{\alpha}^{k}\right) .
$$

Thus, if $u$ and $v$ are such that

$$
\left.u v\right|_{\Omega_{k}} \in \mathbb{P}_{2 N_{k}-1}\left(\Omega_{k}\right),
$$

then

$$
(u, v)_{\Omega_{k}, N_{k}}=\int_{\Omega_{k}} u(\boldsymbol{x}) v(\boldsymbol{x}) \mathrm{d} \boldsymbol{x} .
$$

Similarly, for any continuous vector functions $\boldsymbol{u}$ and $\boldsymbol{v}$, define the scalar product

$$
(\boldsymbol{u}, \boldsymbol{v})_{\Omega_{k}, N_{k}}=\sum_{i=1}^{3}\left(u_{i}, v_{i}\right)_{\Omega_{k}, N_{k}}
$$


It is well known that there exists a constant $C$ such that (see Canuto \& Quarteroni, 1982, see also Remark 13.3 in Bernardi \& Maday, 1994)

$$
\forall \varphi \in \mathbb{P}_{N_{k}}\left(\Omega_{k}\right), \quad\|\varphi\|_{0, \Omega_{k}}^{2} \leqslant(\varphi, \varphi)_{\Omega_{k}, N_{k}} \leqslant C\|\varphi\|_{0, \Omega_{k}}^{2} .
$$

Now, for any multi-index $\eta=\left(n_{1}, \ldots, n_{K}\right) \in \mathbb{N}^{K}$ define $W_{\eta}(\Omega)$ as the space of all the functions $v \in L^{2}(\Omega)$ satisfying

$$
v_{\mid \Omega_{k}} \in \mathbb{P}_{n_{k}}\left(\Omega_{k}\right) .
$$

The global Lagrange interpolation operator $\mathcal{I}_{\delta}$ associated with the grids $\Xi^{k}, k=1, \ldots, K$, is defined as follows: given a function $v$, continuous in each $\Omega_{k}, \mathcal{I}_{\delta} v$ is the only element of $W_{\delta}(\Omega)$ satisfying

$$
\forall k \leqslant K \text { and } \forall \boldsymbol{\xi} \in \Xi^{k}, \quad \mathcal{I}_{\delta} v(\boldsymbol{\xi})=v(\boldsymbol{\xi}) .
$$

The following estimate holds (see Bernardi \& Maday, 1994, Theorem 14.2):

$$
\left\|v-\mathcal{I}_{\delta} v\right\|_{L^{2}(\Omega)} \leqslant c \sum_{k=1}^{K} N_{k}^{-s_{k}}\|v\|_{H^{s_{k}}\left(\Omega_{k}\right)}
$$

and is valid for all $v \in L^{2}(\Omega)$ such that for each $k \leqslant K,\left.v\right|_{\Omega_{k}} \in H^{s_{k}}\left(\Omega_{k}\right)$ for some $s_{k}>3 / 2$.

Now, we are in a position to construct the space $H_{\delta}(\mathbf{c u r l} ; \Omega)$, the discrete counterpart of $H(\mathbf{c u r l} ; \Omega)$. Namely, $H_{\delta}(\mathbf{c u r l} ; \Omega)$ is defined as the space of all the vector functions $v \in W_{\delta}(\Omega)^{3}$ satisfying

$$
\int_{F_{s}^{-}}\left(\boldsymbol{v}^{+} \times \boldsymbol{n}_{s}-\boldsymbol{v}^{-} \times \boldsymbol{n}_{s}\right) \cdot \boldsymbol{\psi} \mathrm{d} \lambda_{2}=0 \quad \forall \boldsymbol{\psi} \in \mathbb{M}_{\delta}^{s}\left(F_{s}^{-}\right),
$$

at each slave face $F_{s}^{-}, s \leqslant m$. Here $\mathbb{M}_{\delta}^{s}\left(F_{s}^{-}\right)$denotes a suitable space of Lagrange multipliers. More precisely, we choose

$$
\forall 1 \leqslant s \leqslant m, \quad \mathbb{M}_{\delta}^{s}\left(F_{s}^{-}\right)=\mathbb{P}_{N_{k}(s)-2}\left(F_{s}^{-}\right)^{2} .
$$

Another adequate choice could be the following

$$
\forall 1 \leqslant s \leqslant m, \quad \mathbb{M}_{\delta}^{s}\left(F_{s}^{-}\right)=\mathbb{P}_{N_{k(s)}-2, N_{k(s)}}\left(F_{s}^{-}\right) \times \mathbb{P}_{N_{k(s)}, N_{k(s)}-2}\left(F_{s}^{-}\right) .
$$

The forthcoming analysis remains valid for both the choices.

Notice that the condition (3.8) is a weaker version of the transmission condition (3.3). Consequently, the inclusion $H_{\delta}($ curl; $\Omega) \hookrightarrow H($ curl; $\Omega)$ does not hold in general.

Finally, define the broken norm $\|\cdot\|_{H_{*}(\operatorname{curl}, \Omega)}$ as follows

$$
\|\boldsymbol{v}\|_{H_{*}(\operatorname{curl}, \Omega)}^{2}=\sum_{k=1}^{K}\left\{(\operatorname{curl} \boldsymbol{v}, \operatorname{curl} v)_{\Omega_{k}}+(\boldsymbol{v}, \boldsymbol{v})_{\Omega_{k}}\right\} .
$$

\subsection{The discrete problem}

Here we wish to approximate the magnetic field $\boldsymbol{H}$ that satisfies the second-order equation

$$
\begin{aligned}
\operatorname{curl}\left(\epsilon^{-1} \operatorname{curl} \boldsymbol{u}\right)+\tau \mu \boldsymbol{u} & =\operatorname{curl}\left(\epsilon^{-1} \boldsymbol{j}\right)+\boldsymbol{u}_{0} & & \text { in } \Omega, \\
\operatorname{curl} \boldsymbol{u} \times \boldsymbol{n} & =\boldsymbol{j} \times \boldsymbol{n} & & \text { on } \partial \Omega,
\end{aligned}
$$


where $\tau>0$ is a given parameter, $\boldsymbol{j}$ is the imposed current source and $\boldsymbol{u}_{0}$ is a data field which comes from time-stepping in the case of time-dependent equations, or from the iteration procedure in the case of time-harmonic regime.

This problem can be written in the weak form: find $\boldsymbol{u} \in H(\mathbf{c u r l} ; \Omega)$ such that

$$
a(\boldsymbol{u}, \boldsymbol{v})=\ell(\boldsymbol{v})
$$

where

$$
\begin{gathered}
a(\boldsymbol{u}, \boldsymbol{v})=\sum_{k=1}^{K}\left\{\int_{\Omega_{k}} \epsilon^{-1} \operatorname{curl} \boldsymbol{u} \cdot \operatorname{curl} \boldsymbol{v} \mathrm{d} \boldsymbol{x}+\tau \int_{\Omega_{k}} \mu \boldsymbol{u} \cdot \boldsymbol{v} \mathrm{d} \boldsymbol{x}\right\}, \\
\ell(\boldsymbol{v})=\sum_{k=1}^{K}\left\{\int_{\Omega_{k}} \epsilon^{-1} \boldsymbol{j} \cdot \operatorname{curl} \boldsymbol{v} \mathrm{d} \boldsymbol{x}+\int_{\Omega_{k}} \boldsymbol{u}_{0} \cdot \boldsymbol{v} \mathrm{d} \boldsymbol{x}\right\} .
\end{gathered}
$$

The corresponding discrete problem is to find $u_{\delta} \in H_{\delta}(\mathbf{c u r l} ; \Omega)$ such that

$$
a_{\delta}\left(\boldsymbol{u}_{\delta}, \boldsymbol{v}_{\delta}\right)=\ell_{\delta}\left(\boldsymbol{v}_{\delta}\right) \quad \forall \boldsymbol{v} \in H_{\delta}(\operatorname{curl} ; \Omega),
$$

with

$$
a_{\delta}\left(\boldsymbol{u}_{\delta}, \boldsymbol{v}_{\delta}\right)=\sum_{k=1}^{K}\left(\epsilon^{-1} \operatorname{curl} \boldsymbol{u}_{\delta}, \operatorname{curl} \boldsymbol{v}_{\delta}\right)_{\Omega_{k}, N_{k}}+\tau\left(\mu \boldsymbol{u}_{\delta}, \boldsymbol{v}_{\delta}\right)_{\Omega_{k}, N_{k}}
$$

and

$$
\ell_{\delta}\left(\boldsymbol{v}_{\delta}\right)=\sum_{m=1}^{K}\left(\epsilon^{-1} \boldsymbol{j}, \operatorname{curl} \boldsymbol{v}_{\delta}\right)_{\Omega_{k}, N_{k}}+\sum_{m=1}^{K}\left(\boldsymbol{u}_{0}, \boldsymbol{v}_{\delta}\right)_{\Omega_{k}, N_{k}} .
$$

Here we supposed that $\epsilon^{-1} \boldsymbol{j}$ and $\boldsymbol{u}_{0}$ are continuous in each $\Omega_{k}$. Observe that there exist two constants $\alpha_{*}\left(\tau, \mu_{0}, \epsilon_{\infty}\right)$ and $\beta_{*}\left(\tau, \mu_{0}, \epsilon_{\infty}\right)$, independent of $\delta$, such that

$$
\begin{aligned}
\forall \boldsymbol{u}_{\delta} \in H_{\delta}(\operatorname{curl} ; \Omega), & a_{\delta}\left(\boldsymbol{u}_{\delta}, \boldsymbol{u}_{\delta}\right) \geqslant \alpha_{*}\left\|\boldsymbol{u}_{\delta}\right\|_{H_{*}(\operatorname{curl}, \Omega)}^{2}, \\
\forall \boldsymbol{u}_{\delta}, \boldsymbol{v}_{\delta} \in H_{\delta}(\operatorname{curl} ; \Omega), & \left|a_{\delta}\left(\boldsymbol{u}_{\delta}, \boldsymbol{v}_{\delta}\right)\right| \leqslant \beta_{*}\left\|\boldsymbol{u}_{\delta}\right\|_{H_{*}(\operatorname{curl}, \Omega)}\left\|\boldsymbol{v}_{\delta}\right\|_{H_{*}(\operatorname{curl}, \Omega)},
\end{aligned}
$$

thanks to (1.4) and (3.6).

REMARK 1 In the case of time-harmonic equations, one could be tempted to consider directly the penalized discrete problem: find $\boldsymbol{u} \in X_{T, \delta}(\mu ; \Omega)$ such that

$$
a_{2, \delta}(\boldsymbol{u}, \boldsymbol{v})=\ell_{2, \delta}(\boldsymbol{v}),
$$

where

$$
a_{2, \delta}(\boldsymbol{u}, \boldsymbol{v})=\sum_{k=1}^{K}\left\{\left(\epsilon^{-1} \operatorname{curl} \boldsymbol{u}, \operatorname{curl} \boldsymbol{v}\right)_{\Omega_{k}, N_{k}}+(\operatorname{div}(\mu \boldsymbol{u}), \operatorname{div}(\mu \boldsymbol{v}))_{\Omega_{k}, N_{k}}-\omega^{2}(\boldsymbol{u}, \boldsymbol{v})_{\Omega_{k}, N_{k}}\right\}
$$

and

$$
\ell_{2, \delta}(v)=\sum_{k=1}^{K}\left(\epsilon^{-1} \boldsymbol{j}, \operatorname{curl} v\right)_{\Omega_{k}, N_{k}}
$$


Here $X_{T, \delta}(\mu ; \Omega)$ denotes the discrete space corresponding to $X_{T}(\mu ; \Omega)$ which is constructed similarly to $H_{\delta}(\mathbf{c u r l} ; \Omega)$ by adding a matching condition on the normal component at the mortars. With such an approach, a natural question arises, whether the semi-norm induced by the semi-inner-product

$$
\sum_{k=1}^{K}\left\{\int_{\Omega_{k}} \epsilon^{-1} \operatorname{curl} \boldsymbol{u} \cdot \operatorname{curl} \boldsymbol{v} \mathrm{d} \boldsymbol{x}+\int_{\Omega_{k}} \operatorname{div}(\mu \boldsymbol{u}) \cdot \operatorname{div}(\mu \boldsymbol{v}) \mathrm{d} \boldsymbol{x}\right\}
$$

may be a uniformly elliptic norm on the discrete space $X_{T, \delta}(\mu ; \Omega)$ (as is the semi-norm (2.3) in the continuous case). The difficulty of this unresolved question and the uncertainty about its affirmative answer are circumvented here by the use of the iterative algorithm proposed in Section 2.2.

\subsection{Error estimate}

The main theoretical result of this paper is the following.

TheOREM 1 Let $\boldsymbol{u} \in H(\mathbf{c u r l} ; \Omega)$ be the solution of (3.12) and $\boldsymbol{u}_{\delta}$ the solution of (3.13). We set

$$
\lambda=\epsilon^{-1}(\operatorname{curl} u-j) .
$$

Suppose that, for each $k \in\{1, \ldots, K\}$,

$$
\boldsymbol{u} \in H^{s_{k}+1}\left(\Omega_{k}\right)^{3}, \quad \lambda \in H^{\sigma_{k}+1}\left(\Omega_{k}\right)^{3}
$$

for some real numbers $s_{k}>1 / 2, \sigma_{k}>1 / 2$. Suppose also that there exists a uniform constant $\eta_{0}$ such that

$$
\eta_{\delta} \leqslant \eta_{0}
$$

Then

$$
\left\|\boldsymbol{u}-\boldsymbol{u}_{\delta}\right\|_{H_{*}(\operatorname{curl}, \Omega)} \leqslant C\left(\sum_{k=1}^{K} N_{k}^{1 / 4-s_{k}}\|\boldsymbol{u}\|_{H^{\delta_{k}+1}\left(\Omega_{k}\right)}+\left(\log N_{k}\right)^{1 / 2} N_{k}^{-\sigma_{k}}\|\boldsymbol{\lambda}\|_{H^{\sigma_{k}+1}\left(\Omega_{k}\right)}\right) .
$$

REMARK 2 The condition (3.17) can be dropped provided that the right-hand side of (3.18) is replaced by

$$
C\left(\left(\eta_{\delta}^{1 / 4}+\eta_{\delta}^{3 / 4}\right) \sum_{k=1}^{K} N_{k}^{1 / 4-s_{k}}\|\boldsymbol{u}\|_{H^{\delta_{k}+1}\left(\Omega_{k}\right)}+\sum_{k=1}^{K} \eta_{\delta}^{\sigma_{k}}\left(\log N_{k}\right)^{1 / 2} N_{k}^{-\sigma_{k}}\|\boldsymbol{\lambda}\|_{H^{\sigma_{k}+1}\left(\Omega_{k}\right)}\right) .
$$

The proof is based on the following lemma.

LEMMA 1 We have

$$
\begin{aligned}
\left\|\boldsymbol{u}-\boldsymbol{u}_{\delta}\right\|_{H_{*}(\operatorname{curl}, \Omega)} \leqslant C & \left\|\boldsymbol{u}-\mathcal{I}_{\delta} \boldsymbol{u}\right\|_{H_{*}(\operatorname{curl}, \Omega)}+\inf _{\boldsymbol{v}_{\delta} \in H_{\delta}(\operatorname{curl} ; \Omega)}\left\|\boldsymbol{u}-\boldsymbol{v}_{\delta}\right\|_{H_{*}(\operatorname{curl}, \Omega)} \\
& +\sup _{\boldsymbol{w}_{\delta} \in H_{\delta}(\operatorname{curl} ; \Omega), w \neq 0} \frac{b\left(\boldsymbol{u}, \boldsymbol{w}_{\delta}\right)-b_{\delta}\left(\boldsymbol{u}, \boldsymbol{w}_{\delta}\right)}{\left\|\boldsymbol{w}_{\delta}\right\|_{H_{*}(\operatorname{curl}, \Omega)}} \\
& +\sup _{\boldsymbol{w}_{\delta} \in H_{\delta}(\operatorname{curl}, \Omega), \boldsymbol{w} \neq 0} \frac{a\left(\boldsymbol{u}, \boldsymbol{w}_{\delta}\right)-\ell\left(\boldsymbol{w}_{\delta}\right)}{\left.\left\|\boldsymbol{w}_{\delta}\right\|_{H_{*}(\operatorname{curl}, \Omega)}\right)}
\end{aligned}
$$

where

$$
b\left(\boldsymbol{u}, \boldsymbol{w}_{\delta}\right)=a\left(\boldsymbol{u}, \boldsymbol{w}_{\delta}\right)-\ell\left(\boldsymbol{w}_{\delta}\right), \quad b_{\delta}\left(\boldsymbol{u}, \boldsymbol{w}_{\delta}\right)=a_{\delta}\left(\mathcal{I}_{\delta} \boldsymbol{u}, \boldsymbol{w}_{\delta}\right)-\ell_{\delta}\left(\boldsymbol{w}_{\delta}\right) .
$$


Note that the last three terms on the right-hand side correspond, respectively, to the best approximation error, the consistency error and the numerical integration error.

Proof. This lemma is a slight modification of the standard Berger-Scott-Strang lemma (see Berger et al., 1972). Indeed, for any $\boldsymbol{v}_{\delta} \in H_{\delta}(\operatorname{curl} ; \Omega)$ one has

$$
\begin{aligned}
\alpha_{*}\left\|\boldsymbol{u}_{\delta}-\boldsymbol{v}_{\delta}\right\|_{H_{*}(\operatorname{curl}, \Omega) \leqslant}^{2} & a_{\delta}\left(\boldsymbol{u}_{\delta}-\boldsymbol{v}_{\delta}, \boldsymbol{u}_{\delta}-\boldsymbol{v}_{\delta}\right)=\ell_{\delta}\left(\boldsymbol{u}_{\delta}-\boldsymbol{v}_{\delta}\right)-a_{\delta}\left(\boldsymbol{v}_{\delta}, \boldsymbol{u}_{\delta}-\boldsymbol{v}_{\delta}\right) \\
\leqslant & \ell_{\delta}\left(\boldsymbol{u}_{\delta}-\boldsymbol{v}_{\delta}\right)+a_{\delta}\left(\mathcal{I}_{\delta} \boldsymbol{u}-\boldsymbol{v}_{\delta}, \boldsymbol{u}_{\delta}-\boldsymbol{v}_{\delta}\right)-a_{\delta}\left(\mathcal{I}_{\delta} \boldsymbol{u}, \boldsymbol{u}_{\delta}-\boldsymbol{v}_{\delta}\right) \\
& +a\left(\boldsymbol{u}, \boldsymbol{u}_{\delta}-\boldsymbol{v}_{\delta}\right)-a\left(\boldsymbol{u}, \boldsymbol{u}_{\delta}-\boldsymbol{v}_{\delta}\right)-\ell\left(\boldsymbol{u}_{\delta}-\boldsymbol{v}_{\delta}\right)+\ell\left(\boldsymbol{u}_{\delta}-\boldsymbol{v}_{\delta}\right) .
\end{aligned}
$$

Hence,

$$
\begin{aligned}
\alpha_{*}\left\|\boldsymbol{u}_{\delta}-\boldsymbol{v}_{\delta}\right\|_{H_{*}(\operatorname{curl}, \Omega)} \leqslant & \beta_{*}\left\|\boldsymbol{I}_{\delta} \boldsymbol{u}-\boldsymbol{v}_{\delta}\right\|_{H_{*}(\operatorname{curl}, \Omega)} \\
& +\sup _{\boldsymbol{w}_{\delta} \in H_{\delta}(\operatorname{curl} ; \Omega), \boldsymbol{w}_{\delta} \neq \mathbf{0}} \frac{\left|b\left(\boldsymbol{u}, \boldsymbol{w}_{\delta}\right)-b_{\delta}\left(\boldsymbol{u}, \boldsymbol{w}_{\delta}\right)\right|}{\left\|\boldsymbol{w}_{\delta}\right\|_{H_{*}(\operatorname{curl}, \Omega)},} \\
& +\sup _{\boldsymbol{w}_{\delta} \in H_{\delta}(\operatorname{curl} ; \Omega), \boldsymbol{w}_{\delta} \neq \mathbf{0}} \frac{\left|a\left(\boldsymbol{u}, \boldsymbol{w}_{\delta}\right)-\ell\left(\boldsymbol{w}_{\delta}\right)\right|}{\left\|\boldsymbol{w}_{\delta}\right\|_{H_{*}(\operatorname{curl}, \Omega)}} .
\end{aligned}
$$

Finally, using the inequalities

$$
\begin{aligned}
\left\|\boldsymbol{u}-\boldsymbol{u}_{\delta}\right\|_{H_{*}(\operatorname{curl}, \Omega)} & \leqslant\left\|\boldsymbol{u}-\boldsymbol{v}_{\delta}\right\|_{H_{*}(\operatorname{curl}, \Omega)}+\left\|\boldsymbol{u}_{\delta}-\boldsymbol{v}_{\delta}\right\|_{H_{*}(\operatorname{curl}, \Omega)}, \\
\left\|\mathcal{I}_{\delta} \boldsymbol{u}-\boldsymbol{v}_{\delta}\right\|_{H_{*}(\operatorname{curl}, \Omega)} & \leqslant\left\|\boldsymbol{u}-\mathcal{I}_{\delta} \boldsymbol{u}\right\|_{H_{*}(\operatorname{curl}, \Omega)}+\left\|\boldsymbol{u}-\boldsymbol{v}_{\delta}\right\|_{H_{*}(\operatorname{curl}, \Omega)},
\end{aligned}
$$

completes the proof of (3.19).

The rest of this section is devoted to the analysis of each term on the right-hand side of (3.18).

3.3.1 The interpolation and the best approximation error. The estimate of the best approximation error consists of finding a good interpolant of $\boldsymbol{u}$ in the discrete space $H_{\delta}(\mathbf{c u r l} ; \Omega)$. The construction of such an approximation constitutes one of the main difficulties in the numerical analysis of the mortar method.

Proposition 2 Suppose that

$$
\left.\boldsymbol{u}\right|_{\Omega_{k}} \in H^{s_{k}+1}\left(\Omega_{k}\right)
$$

for some real numbers $s_{k}>3 / 2(k=1, \ldots, K)$. Suppose also that the assumption (3.17) is satisfied. Then

$$
\left\|\boldsymbol{u}-\mathcal{I}_{\delta} \boldsymbol{u}\right\|_{H_{*}(\operatorname{curl}, \Omega)}+\inf _{\boldsymbol{v}_{\delta} \in H_{\delta}(\operatorname{curl} ; \Omega)}\left\|\boldsymbol{u}-\boldsymbol{v}_{\delta}\right\|_{H_{*}(\operatorname{curl}, \Omega)} \leqslant c \sum_{k=1}^{K} N_{k}^{1 / 4-s_{k}}\|\boldsymbol{u}\|_{H^{s_{k}+1}\left(\Omega_{k}\right)},
$$

where $c$ is not depending either on $\delta$ or on $\boldsymbol{u}$.

The proof of Proposition 2 is divided into several steps. Let us start by introducing some useful notations. Let $\Lambda=]-1,1\left[{ }^{3}\right.$ be the unit cube and $\left.F=\right]-1,1\left[{ }^{2} \times\{1\}\right.$ its upper face. Let $\boldsymbol{e}_{1}=(1,0,0)$, $\boldsymbol{e}_{2}=(0,1,0)$ and $\boldsymbol{e}_{3}=(0,0,1)$ be the canonical basis of $\mathbb{R}^{3}$. Consider a collection of non-trivial open 
rectangles $G_{1}, \ldots, G_{r}$ such that

- $G_{i} \cap G_{j}=\emptyset$ for any $i, j$ such that $i \neq j$,

- $\bar{F}=\bigcup_{i=1}^{r} \bar{G}_{i}$.

- The edges of each $G_{k}$ are parallel to the co-ordinate axes.

Denote by $\widetilde{H}^{1}(F)$ the space of all the functions $u \in L^{2}(F)$ such that

$$
u_{\mid G_{i}} \in H^{1}\left(G_{i}\right) \text { for all } i \in\{1, \ldots, r\} .
$$

\section{Step 1: some discrete operators}

For any integers $N$ and $M, \pi_{N, M}$ stands for the $L^{2}$ projector from $L^{2}(F)$ onto $\mathbb{P}_{N, M}(F)$. We denote by $\mathbb{P}_{N, M}^{*, x}(F)$ (resp. $\mathbb{P}_{N, M}^{*, y}(F)$ ) the space of all the polynomial functions $v \in \mathbb{P}_{N, M}(F)$ satisfying $v=0$

at $x= \pm 1$ (resp. at $y= \pm 1$ ). Define also the operator $\pi_{N, M}^{*, x}$ as follows: for all $u \in L^{2}(\Omega), \pi_{N, M}^{*, x} u$ is the unique element of $\mathbb{P}_{N, M}^{*, x}(F)$ satisfying

$$
\int_{F}\left(u-\pi_{N, M}^{*, x} u\right) \cdot \psi \mathrm{d} x \mathrm{~d} y=0, \quad \text { for any } \psi \in \mathbb{P}_{N-2, M}(F) .
$$

Similarly, let us introduce the operator $\pi_{N, M}^{*, y}$ defined as: for all $u \in L^{2}(\Omega), \pi_{N, M}^{*, y} u$ is the unique element of $\mathbb{P}_{N, M}^{*, y}(F)$ such that

$$
\int_{F}\left(u-\pi_{N, M}^{*, y} u\right) \cdot \psi \mathrm{d} x \mathrm{~d} y=0, \quad \text { for any } \psi \in \mathbb{P}_{N, M-2}(F) .
$$

We state the following result.

Proposition 3 Let $N \geqslant 1$ and $M \geqslant 1$ be two integers. There exist two constants $c_{1}, c_{2}$, depending neither on $N$ nor on $M$, such that

$$
\begin{gathered}
\left\|\pi_{N, M}^{*, x} u\right\|_{L^{2}(F)} \leqslant c_{1} N^{-1} \sum_{k=1}^{r}\left(\|u\|_{H^{1}\left(G_{k}\right)}+N\|u\|_{L^{2}\left(G_{k}\right)}\right), \\
\left\|\frac{\partial\left(\pi_{N, M}^{*, x} u\right)}{\partial x}\right\|_{L^{2}(F)} \leqslant c_{2} N^{1 / 2} \sum_{k=1}^{r}\left(\|u\|_{H^{1}\left(G_{k}\right)}+N\|u\|_{L^{2}\left(G_{k}\right)}\right),
\end{gathered}
$$

for each $u \in \widetilde{H}^{1}(F)$. Similar estimates hold for the operator $\pi_{N, M}^{*, y}$ with $N$ replaced by $M$ and $x$ replaced by $y$.

Proof. The following lemma is a first step towards the proof of Proposition 3.

LEMma 2 Let $N \geqslant 1$ and $M \geqslant 1$ be two integers. There exists a constant $c$ not depending either on $N$ or on $M$ such that for any function $u \in \widetilde{H}^{1}(F)$ one has

$$
\left\|\left(\pi_{N, M} u\right)( \pm 1, \cdot)\right\|_{L^{2}(]-1,1[)} \leqslant c_{1} N^{-1 / 2} \sum_{k=1}^{r}\left(\|u\|_{H^{1}\left(G_{k}\right)}+N\|u\|_{L^{2}\left(G_{k}\right)}\right) .
$$


Proof. Let $u \in \widetilde{H}^{1}(F)$. We set $w=\pi_{N, M} u \in \mathbb{P}_{N, M}(F)$ and

$$
q_{N}(x, y)=\left(L_{N}(x)+L_{N+1}(x)\right) w(1, y) \in \mathbb{P}_{N+1, M}(F) .
$$

We have on the one hand

$$
\int_{-1}^{1} w(x, y) \frac{\partial q_{N}}{\partial x}(x, y) \mathrm{d} x=2 w(1, y)^{2}
$$

since $q_{N}$ is orthogonal to the space $\mathbb{P}_{N-1, M}(F)$. On the other hand, we can write

$$
\int_{F} w(x, y) \frac{\partial q_{N}}{\partial x}(x, y) \mathrm{d} x \mathrm{~d} y=\int_{F} u(x, y) \frac{\partial q_{N}}{\partial x}(x, y) \mathrm{d} x \mathrm{~d} y .
$$

Hence,

$$
\begin{aligned}
2 \int_{-1}^{1} w^{2}(1, y) \mathrm{d} y & =\sum_{k=1}^{r} \int_{G_{k}} u(x, y) \frac{\partial q_{N}}{\partial x}(x, y) \mathrm{d} x \mathrm{~d} y \\
& =\sum_{k=1}^{r}\left(-\int_{G_{k}} \frac{\partial u}{\partial x}(x, y) q_{N}(x, y) \mathrm{d} x \mathrm{~d} y+\int_{\partial G_{k}} u(x, y) q_{N}(x, y) \boldsymbol{n} \cdot \boldsymbol{e}_{1} \mathrm{~d} \sigma\right) \\
& \leqslant c \sum_{k=1}^{r}\left(N^{-1 / 2}\left\|\frac{\partial u}{\partial x}\right\|_{L^{2}\left(G_{k}\right)}+\|u\|_{L^{2}\left(\partial G_{k}\right)}\right)\|w(1, \cdot)\|_{\left.\left.L^{2}(]-1,1\right]\right)},
\end{aligned}
$$

where we used the Cauchy-Schwarz inequality combined with the estimates

$$
\begin{aligned}
\left\|q_{N}\right\|_{L^{2}(F)}^{2} & =\left(\left\|L_{N}\right\|_{L^{2}(]-1,1[)}^{2}+\left\|L_{N+1}\right\|_{L^{2}(]-1,1[)}^{2}\right)\|w(1, \cdot)\|_{L^{2}(]-1,1[)}^{2} \\
& \leqslant C N^{-1}\|w(1, \cdot)\|_{L^{2}(]-1,1[)}^{2} \\
|q(x, y)| & \leqslant\left(\max _{-1 \leqslant x \leqslant 1}\left|L_{N-1}(x)\right|+\max _{-1 \leqslant x \leqslant 1}\left|L_{N-2}(x)\right|\right)|w(1, y)| \\
& \leqslant 2|w(1, y)| \quad \forall(x, y) \in F .
\end{aligned}
$$

Recall that there exists a constant $C$ such that for any $\epsilon>0$ the inequality

$$
\|v\|_{L^{2}\left(\partial G_{k}\right)} \leqslant c\left(\epsilon\|v\|_{H^{1}\left(G_{k}\right)}+\epsilon^{-1}\|v\|_{L^{2}\left(G_{k}\right)}\right)
$$

holds for any $v \in H^{1}\left(G_{k}\right)$. Taking $v=u$ and $\epsilon=N^{-1 / 2}$ gives (3.25).

We need also the following lemma.

LEMma 3 There exists a constant $c$ such that for any integers $N$ and $M$ and any polynomial function $p$ in $\mathbb{P}_{N, M}(]-1,1\left[^{2}\right)$, the following inequality holds

$$
\|p\|_{L^{2}(T)} \leqslant C N\|p\|_{L^{2}(]-1,1\left[^{2}\right)}
$$

where $T=(\{-1\} \times]-1,1[) \cup(\{1\} \times]-1,1[)$.

Proof. Let $p$ be in $\mathbb{P}_{N, M}(]-1,1\left[^{2}\right)$. We write its expansion in the basis of Legendre polynomials

$$
p(x, y)=\sum_{k=0}^{N} \sum_{j=0}^{M} a_{k, j} L_{k}(x) L_{j}(y),
$$


so that

$$
\|p\|_{L^{2}(]-1,1\left[^{2}\right)}^{2}=\sum_{k=0}^{N} \sum_{j=0}^{M} \frac{4 a_{k, j}^{2}}{(2 k+1)(2 j+1)} .
$$

On the other hand, we have

$$
\begin{aligned}
\|p\|_{L^{2}(T)}^{2} & =\left\|\sum_{j=0}^{M}\left(\sum_{k=0}^{N}(-1)^{k} a_{k, j}\right) L_{j}(\cdot)\right\|_{L^{2}(]-1,1[)}^{2}+\left\|\sum_{j=0}^{M}\left(\sum_{k=0}^{N} a_{k, j}\right) L_{j}(\cdot)\right\|_{L^{2}(]-1,1[)}^{2} \\
& =\sum_{j=0}^{M} \frac{2\left(\sum_{k=0}^{N}(-1)^{k} a_{k, j}\right)^{2}}{2 j+1}+\sum_{j=0}^{M} \frac{2\left(\sum_{k=0}^{N} a_{k, j}\right)^{2}}{2 j+1}
\end{aligned}
$$

Then, using the Cauchy-Schwarz inequality leads to

$$
\begin{aligned}
\|p\|_{L^{2}(T)}^{2} & =4\left(\sum_{k=0}^{N}(2 k+1)\right) \sum_{j=0}^{M} \sum_{k=0}^{N} \frac{a_{k, j}^{2}}{(2 k+1)(2 j+1)} \\
& \leqslant C N^{2}\|p\|_{L^{2}(]-1,1\left[^{2}\right)^{\circ}}^{2}
\end{aligned}
$$

This completes the proof of Lemma 3.

Now, we are in position to prove Proposition 3. Let $u \in \widetilde{H}^{1}(F)$ and set $w=\pi_{N-2, M} u \in \mathbb{P}_{N, M}(F)$ and $v=\pi_{N, M}^{*, x} u$. We prove easily that

$$
v(x, y)=w(x, y)-\frac{1}{2}\left(L_{N-1}(x)+L_{N}(x)\right) w(1, y)-\frac{1}{2}\left(L_{N-1}(-x)+L_{N}(-x)\right) w(-1, y) .
$$

Thus,

$$
\|v\|_{L^{2}(F)} \leqslant\|w\|_{L^{2}(F)}+\frac{C}{N^{1 / 2}}\left(\|w(1, \cdot)\|_{L^{2}(]-1,1[)}+\|w(-1, \cdot)\|_{\left.\left.L^{2}(]-1,1\right]\right)}\right) .
$$

Using (3.25) yields (3.23).

On the other hand, one has

$$
\begin{aligned}
\int_{F}\left(\frac{\partial v}{\partial x}\right)^{2} \mathrm{~d} x \mathrm{~d} y & =-\int_{F} v \frac{\partial^{2} v}{\partial x^{2}} \mathrm{~d} x \mathrm{~d} y \\
& =-\int_{F} u \frac{\partial^{2} v}{\partial x^{2}} \mathrm{~d} x \mathrm{~d} y \\
& =\sum_{k=1}^{r} \int_{G_{k}} \frac{\partial u}{\partial x} \frac{\partial v}{\partial x} \mathrm{~d} x \mathrm{~d} y-\int_{\partial G_{k}} u \frac{\partial v}{\partial x} \boldsymbol{n} \cdot \boldsymbol{e}_{x} \mathrm{~d} \sigma \\
& \leqslant \sum_{k=1}^{r}\left(\|u\|_{H^{1}\left(G_{k}\right)}+N\|u\|_{L^{2}\left(\partial G_{k}\right)}\right)\left\|\frac{\partial v}{\partial x}\right\|_{L^{2}\left(G_{k}\right)},
\end{aligned}
$$

where we used the inverse inequality

$$
\left\|\frac{\partial v}{\partial x} \boldsymbol{n} \cdot \boldsymbol{e}_{x}\right\|_{L^{2}\left(\partial G_{k}\right)} \leqslant C N\left\|\frac{\partial v}{\partial x}\right\|_{L^{2}\left(G_{k}\right)},
$$

which follows from Lemma 3. This ends the proof of Proposition 3. 


\section{Step 2: a lifting operator}

Lemma 4 Let $N \geqslant 1$ and $M \geqslant 1$ be two integers. Then, for all $v \in \mathbb{P}_{N, M}^{*, x}(F)$ there exists a function $\psi \equiv \mathcal{R}_{N, M}^{y} v \in \mathbb{P}_{N, M, N}(\Lambda)$ such that

$$
\begin{aligned}
\psi & =v, & & \text { on } F, \\
\psi \boldsymbol{e}_{2} \times \boldsymbol{n} & =\mathbf{0}, & & \text { on } \partial \Lambda-F .
\end{aligned}
$$

Moreover, there exists a constant $c$ not depending either on $N$ or on $M$ such that

$$
\left\|\psi \boldsymbol{e}_{2}\right\|_{H(\mathbf{c u r l} ; A)}^{2} \leqslant c\|v\|_{L^{2}(F)}\left\|\frac{\partial v}{\partial x}\right\|_{L^{2}(F)} .
$$

Proof. In the following, $c$ and $C$ denote two generic constants not depending on $N$ and $M$. We set

$$
\psi(x, y, z)=\varphi_{N}(z) v(x, y),
$$

where $\varphi_{N} \in \mathbb{P}_{N}(]-1,1[)$ is a function which satisfies

$$
\varphi_{N}(1)=1, \quad \varphi_{N}(-1)=0,
$$

and which will be chosen later. It is quite clear that $\psi$ fulfills (3.27). On the other hand, we have

$$
\begin{gathered}
\|\psi\|_{L^{2}(A)}^{2}=\left\|\varphi_{N}\right\|_{\left.\left.L^{2}(]-1,1\right]\right)}^{2}\|v\|_{L^{2}(F)}^{2}, \\
\left\|\operatorname{curl}\left(\psi \boldsymbol{e}_{2}\right)\right\|_{L^{2}(A)}^{2}=\left\|\varphi_{N}\right\|_{\left.\left.L^{2}(]-1,1\right]\right)}^{2}\left\|\frac{\partial v}{\partial x}\right\|_{L^{2}(F)}^{2}+\|v\|_{L^{2}(F)}^{2}\left\|\varphi_{N}^{\prime}\right\|_{L^{2}(]-1,1[)}^{2}
\end{gathered}
$$

Thus, if $v \neq 0$

$$
\left\|\operatorname{curl}\left(\psi \boldsymbol{e}_{2}\right)\right\|_{L^{2}(\Lambda)}^{2}=\left(\left\|\varphi_{N}\right\|_{L^{2}(]-1,1[)}^{2} \omega_{v}^{2}+\left\|\varphi_{N}^{\prime}\right\|_{L^{2}(]-1,1[)}^{2}\right)\|v\|_{L^{2}(F)}^{2},
$$

where

$$
\omega_{v}=\frac{\left\|\frac{\partial v}{\partial x}\right\|_{L^{2}(F)}}{\|v\|_{L^{2}(F)}} .
$$

Note that

$$
c \leqslant \omega_{v} \leqslant C N^{2}
$$

thanks to the inverse inequality

$$
\left\|\frac{\partial v}{\partial x}\right\|_{L^{2}(F)} \leqslant C N^{2}\|v\|_{L^{2}(F)},
$$

and the Poincaré inequality

$$
\left\|\frac{\partial v}{\partial x}\right\|_{L^{2}(F)} \geqslant c\|v\|_{L^{2}(F)} .
$$

Now, we choose

$$
\varphi_{N}(z)=\alpha p_{N}(z)+\beta p_{N+1}(z),
$$


where for any $m \in\{N, N+1\}, p_{m}(z)$ is defined as

$$
p_{m}(z)=\sum_{k \geqslant 0} \frac{1}{\omega_{v}^{2(k+1)}} L_{m}^{(2 k+1)}(z),
$$

while $\alpha$ and $\beta$ are given by

$$
\alpha=\frac{1}{2 p_{N}(1)}, \quad \beta=\frac{1}{2 p_{N+1}(1)} .
$$

Observe that

$$
|\alpha| \leqslant c \omega_{v}, \quad|\beta| \leqslant c \omega_{v},
$$

since

$$
p_{m}(1) \geqslant \frac{L_{m}^{\prime}(1)}{\omega_{v}^{2}} \geqslant \frac{c m^{2}}{\omega_{v}^{2}} \geqslant \frac{c}{\omega_{v}},
$$

for $m=N$ or $m=N+1$ (thanks to (3.30)). Observe also that $\varphi_{N}$ satisfies

$$
\varphi_{N}(1)=1, \quad \varphi_{N}(-1)=0
$$

and

$$
\omega_{v}^{2} \varphi_{N}(z)-\varphi_{N}^{\prime \prime}(z)=\alpha L_{N}^{\prime}(z)+\beta L_{N+1}^{\prime}(z) .
$$

Multiplying the latter by $\varphi_{N}(z)$ and integrating by parts on ] $-1,1$ [ gives

$$
\omega_{v}^{2}\left\|\varphi_{N}\right\|_{L^{2}(]-1,1[)}^{2}+\left\|\varphi_{N}^{\prime}\right\|_{L^{2}(]-1,1[)}^{2}=\lambda_{N}
$$

where $\lambda_{N}=\varphi_{N}^{\prime}(1)+\alpha+\beta$.

On the other hand, multiplying $(3.33)$ by $2(z+1) \varphi_{N}^{\prime}(z)$ and integrating by parts again yields $\omega_{v}^{2}\left\|\varphi_{N}\right\|_{L^{2}(]-1,1[)}^{2}-\left\|\varphi_{N}^{\prime}\right\|_{L^{2}(]-1,1[)}^{2}=2 \omega_{v}^{2}-2 \varphi_{N}^{\prime}(1)^{2}-4(\alpha+\beta) \varphi_{N}^{\prime}(1)=2 \omega_{v}^{2}-2 \lambda_{N}^{2}+2(\alpha+\beta)^{2}$. Hence,

$$
2 \omega_{v}^{2}\left\|\varphi_{N}\right\|_{L^{2}(]-1,1[)}^{2}=-2 \lambda_{N}^{2}+\lambda_{N}+2 \omega_{v}^{2}+2(\alpha+\beta)^{2} \geqslant 0
$$

and

$$
2\left\|\varphi_{N}^{\prime}\right\|_{L^{2}(]-1,1[)}^{2}=2 \lambda_{N}^{2}+\lambda_{N}-2 \omega_{v}^{2}-2(\alpha+\beta)^{2} \geqslant 0 .
$$

It follows that

$$
\frac{-1+\sqrt{1+16 \omega_{v}^{2}+16(\alpha+\beta)^{2}}}{4} \leqslant \lambda_{N} \leqslant \frac{1+\sqrt{1+16 \omega_{v}^{2}+16(\alpha+\beta)^{2}}}{4} \leqslant C \omega_{v} .
$$

Finally, we get after substitution into (3.29)

$$
\begin{gathered}
\left\|\operatorname{curl}\left(\psi \boldsymbol{e}_{2}\right)\right\|_{L^{2}(\Lambda)}^{2} \leqslant c\left\|\frac{\partial v}{\partial x}\right\|_{L^{2}(F)}\|v\|_{L^{2}(F)}, \\
\|\psi\|_{L^{2}(\Lambda)}^{2} \leqslant \frac{c}{\omega_{v}}\|v\|_{L^{2}(F)}^{2}, \\
\leqslant \frac{\|v\|_{L^{2}(F)}^{3}}{\left\|\frac{\partial v}{\partial x}\right\|_{L^{2}(F)}} \\
\leqslant c\|v\|_{L^{2}(F)}\left\|\frac{\partial v}{\partial x}\right\|_{L^{2}(F)}
\end{gathered}
$$

where we used Poincaré's inequality (3.32). Inequality (3.28) follows immediately. 
REMARK 3 The choice of the polynomial $\varphi_{N}$ is not as arbitrary as it seems. Indeed it minimizes the functional

$$
\omega_{v}^{2}\left\|\varphi_{N}\right\|_{L^{2}(]-1,1[)}^{2}+\left\|\varphi_{N}^{\prime}\right\|_{L^{2}(]-1,1[)}^{2},
$$

on the set of all the polynomial functions $p \in \mathbb{P}_{N}(]-1,1[)$ satisfying the conditions $p(1)=1$ and $p(-1)=0$.

Proposition 4 Let $N \geqslant 1$ and $M \geqslant 1$ be two integers. Then, there exists an operator $\boldsymbol{\mathcal { R }}_{N, M}$ from $L^{2}(\Lambda)^{3}$ onto $\mathbb{P}_{N, M, M}(\Lambda) \times \mathbb{P}_{N, M, N}(\Lambda) \times\{0\}$ such that for all $\boldsymbol{u} \in L^{2}(F)^{3}, \boldsymbol{\mathcal { R }}_{N, M} \boldsymbol{u}$ satisfies

$$
\begin{array}{rlrl}
\int_{F}\left(\mathcal{R}_{N, M} \boldsymbol{u} \times \boldsymbol{n}\right) \cdot \boldsymbol{\psi} \mathrm{d} x \mathrm{~d} y & =\int_{F}(\boldsymbol{u} \times \boldsymbol{n}) \cdot \boldsymbol{\psi} \mathrm{d} x \mathrm{~d} y, & & \text { for any } \boldsymbol{\psi} \in \mathbb{P}_{N-2, M}(F) \times \mathbb{P}_{N, M-2}(F), \\
\mathcal{R}_{N, M} \boldsymbol{u} \times \boldsymbol{n}=\mathbf{0}, & & \text { on } \partial \Lambda-F .
\end{array}
$$

Moreover, there exists a constant $c$, independent of $N$ and $M$, such that for all $\boldsymbol{u}=\left(u_{1}, u_{2}, u_{3}\right) \in$ $\widetilde{H}(F)^{2} \times L^{2}(F)$, we have

$$
\begin{aligned}
\left\|\boldsymbol{R}_{N, M} \boldsymbol{u}\right\|_{H(\operatorname{curl} ; A)} \leqslant c & \left\{M^{-1 / 4} \sum_{k=1}^{r}\left(\left\|u_{1}\right\|_{H^{1}\left(G_{k}\right)}+M\left\|u_{1}\right\|_{L^{2}\left(G_{k}\right)}\right)\right. \\
& \left.+N^{-1 / 4} \sum_{k=1}^{r}\left(\left\|u_{2}\right\|_{H^{1}\left(G_{k}\right)}+N\left\|u_{2}\right\|_{L^{2}\left(G_{k}\right)}\right)\right\} .
\end{aligned}
$$

Proof. Let $\boldsymbol{u}=\left(u_{1}, u_{2}, u_{3}\right) \in L^{2}(F)^{3}$ and set

$$
\boldsymbol{\mathcal { R }}_{N, M} \boldsymbol{u}=w_{1} \boldsymbol{e}_{1}+w_{2} \boldsymbol{e}_{2},
$$

where $w_{1}=\left(\mathcal{R}_{N, M}^{x} \circ \pi_{N, M}^{*, y}\right) u_{1}$ and $w_{2}=\left(\mathcal{R}_{N, M}^{y} \circ \pi_{N, M}^{*, x}\right) u_{2}$. Here $\mathcal{R}_{N, M}^{y}$ denotes the lifting operator of Lemma 4, while $\mathcal{R}_{N, M}^{x}$ is the operator obtained from $\mathcal{R}_{N, M}^{y}$ by exchanging $x$ and $y$. It is quite clear that $\boldsymbol{\mathcal { R }}_{N, M} \boldsymbol{u}$ satisfies (3.34). Furthermore, we have

$$
\begin{aligned}
&\left\|\boldsymbol{\mathcal { R }}_{N, M} \boldsymbol{u}\right\|_{H(\mathbf{c u r l}, \Lambda)}^{2} \leqslant c(\left\|\pi_{N, M}^{*, y} u_{1}\right\|_{L^{2}(F)}\left\|\frac{\partial\left(\pi_{N, M}^{*, y} u_{1}\right)}{\partial y}\right\|_{L^{2}(F)} \\
&\left.+\left\|\pi_{N, M}^{*, x} u_{2}\right\|_{L^{2}(F)}\left\|\frac{\partial\left(\pi_{N, M}^{*, x} u_{2}\right)}{\partial x}\right\|_{L^{2}(F)}\right) \\
& \leqslant c\left(M^{-1 / 2}\left\{\sum_{k=1}^{r}\left(\left\|u_{1}\right\|_{H^{1}\left(G_{k}\right)}+M\left\|u_{1}\right\|_{L^{2}\left(G_{k}\right)}\right)\right\}^{2}\right. \\
&\left.+N^{-1 / 2}\left\{\sum_{k=1}^{r}\left(\left\|u_{2}\right\|_{H^{1}\left(G_{k}\right)}+N\left\|u_{2}\right\|_{L^{2}\left(G_{k}\right)}\right)\right\}^{2}\right),
\end{aligned}
$$

which is the desired result. 


\section{Step 3: proof of Proposition 2}

Let us now find an element $\widetilde{\boldsymbol{u}}_{\delta}$ in the discrete space $H_{\delta}(\operatorname{curl} ; \Omega)$ which is a good interpolant of $\boldsymbol{u}$. Firstly, we put

$$
\widetilde{\boldsymbol{u}}_{\delta}=\mathcal{I}_{\delta} \boldsymbol{u}+\boldsymbol{w}_{\delta}
$$

where $\boldsymbol{w}_{\delta}$ will be chosen subsequently. Let $\boldsymbol{z}$ be the function defined on the skeleton $\mathcal{S}$ as follows

$$
z=\left[\mathcal{I}_{\delta} \boldsymbol{u}-\boldsymbol{u}\right]=\left[\mathcal{I}_{\delta} \boldsymbol{u}\right] \text { on } F_{s}^{-}, \quad \text { for } s=1, \ldots, m .
$$

Let $F_{s}^{-}, s \leqslant m$, be a mortar face. For any $\ell \in \mathbb{E}(s)$ we set $\Gamma_{s, \ell}=F_{s}^{-} \cap \partial \Omega_{\ell}$ and we define the following extension of $z$

$$
\mathcal{R}_{s} z= \begin{cases}\mathcal{R}_{M_{s}} z, & \text { in } \Omega_{k(s)}, \\ \mathbf{0}, & \text { elsewhere, }\end{cases}
$$

where $\boldsymbol{R}_{M_{s}}$ is the lifting operator in $\Omega_{k(s)}$ constructed from the operator $\boldsymbol{R}_{M_{s}, M_{s}}$ of Proposition 4 by translation, homothety and rotation.

It follows from Proposition 4 that

$$
\begin{aligned}
\left\|\boldsymbol{\mathcal { R }}_{s} z\right\|_{H_{*}(\mathbf{c u r l}, \Omega)} & \leqslant c M_{s}^{-1 / 4} \sum_{\ell \in \widetilde{\mathbb{E}}(s)}\left(\left\|z \times \boldsymbol{n}_{s}\right\|_{H^{1}\left(F_{s}^{-} \cap \partial \Omega_{\ell}\right)}+M_{S}\left\|\boldsymbol{z} \times \boldsymbol{n}_{s}\right\|_{L^{2}\left(F_{s}^{-} \cap \partial \Omega_{\ell}\right)}\right) \\
& \leqslant c M_{s}^{-1 / 4} \sum_{\ell \in \mathbb{E}(s)}\left(\left\|\left(\mathcal{I}_{\delta} \boldsymbol{u}-\boldsymbol{u}\right) \times \boldsymbol{n}_{s}\right\|_{H^{1}\left(F_{s}^{-} \cap \partial \Omega_{\ell}\right)}+M_{s}\left\|\left(\mathcal{I}_{\delta} \boldsymbol{u}-\boldsymbol{u}\right) \times \boldsymbol{n}_{s}\right\|_{L^{2}\left(F_{s}^{-} \cap \partial \Omega_{\ell}\right)}\right) \\
& \left.\leqslant c M_{S}^{-1 / 4} \sum_{\ell \in \mathbb{E}(s)}\left(N_{\ell}^{1 / 2-s_{\ell}}+M_{S} N_{\ell}^{-1 / 2-s_{\ell}}\right)\|\boldsymbol{u} \times \boldsymbol{n}\|_{H^{1 / 2+s} \ell\left(\partial \Omega_{\ell}\right)}\right) \\
& \leqslant c\left(\eta_{\delta}^{1 / 4}+\eta_{\delta}^{3 / 4}\right) \sum_{k=1}^{K} N_{k}^{1 / 4-s_{k}}\|\boldsymbol{u}\|_{H^{s_{k}+1}\left(\Omega_{k}\right)} .
\end{aligned}
$$

Finally, we choose

$$
\boldsymbol{w}_{\delta}=\sum_{s=1}^{m} \boldsymbol{R}_{s} \boldsymbol{z}
$$

Then,

$$
\begin{aligned}
\left\|\boldsymbol{u}-\widetilde{\boldsymbol{u}}_{\delta}\right\|_{H_{*}(\operatorname{curl}, \Omega)} & \leqslant\left\|\boldsymbol{u}-\boldsymbol{I}_{\delta} \boldsymbol{u}\right\|+\sum_{s=1}^{m}\left\|\boldsymbol{\mathcal { R }}_{s} z\right\|_{H_{*}(\operatorname{curl}, \Omega)} \\
& \leqslant C\left(\eta_{\delta}^{1 / 4}+\eta_{\delta}^{3 / 4}\right) \sum_{k=1}^{K} N_{k}^{1 / 4-s_{k}}\|\boldsymbol{u}\|_{H^{s}+1}\left(\Omega_{k}\right)
\end{aligned}
$$

Using (3.17) ends the proof of (3.20).

3.3.2 The consistency error. Let $\lambda$ be the vector field defined by (3.16). Note that $\lambda \in H_{0}(\operatorname{curl} ; \Omega)$ since

$$
\operatorname{curl} \boldsymbol{\lambda}=-\tau \mu \boldsymbol{u}+\boldsymbol{u}_{0} \text { in } \Omega, \quad \boldsymbol{\lambda} \times \boldsymbol{n}=\mathbf{0} \text { on } \partial \Omega .
$$

An estimate of the consistency error in terms of the vector field $\boldsymbol{\lambda}$ is given in the following. 
Proposition 5 Assume that for each $k \in\{1, \ldots, K\},\left.\lambda\right|_{\Omega_{k}} \in H^{\sigma_{k}+1}\left(\Omega_{k}\right)$ for some real $\sigma_{k}>1 / 2$. Then

$$
\sup _{\boldsymbol{w}_{\delta} \in H_{\delta}(\mathbf{c u r l} ; \Omega), \boldsymbol{w}_{\delta} \neq 0} \frac{a\left(\boldsymbol{u}, \boldsymbol{w}_{\delta}\right)-\ell\left(\boldsymbol{w}_{\delta}\right)}{\left\|\boldsymbol{w}_{\delta}\right\|_{H_{*}(\operatorname{curl}, \Omega)}} \leqslant c \sum_{k=0}^{K} \eta_{\delta}^{\sigma_{k}}\left(\log N_{k}\right)^{1 / 2} N_{k}^{-\sigma_{k}}\|\boldsymbol{\lambda}\|_{H^{\sigma_{k}+1}\left(\Omega_{k}\right)} .
$$

Proof. Observe that

$$
\begin{aligned}
a\left(\boldsymbol{u}, \boldsymbol{w}_{\delta}\right)-\ell\left(\boldsymbol{w}_{\delta}\right) & =\sum_{k=1}^{K}\left\{\left(\epsilon^{-1} \operatorname{curl} \boldsymbol{u}, \operatorname{curl} \boldsymbol{w}_{\delta}\right)_{\Omega_{k}}+\tau\left(\mu \boldsymbol{u}, \boldsymbol{w}_{\delta}\right)_{\Omega_{k}}-\left(\epsilon^{-1} \boldsymbol{j}, \operatorname{curl} \boldsymbol{w}_{\delta}\right)_{\Omega_{k}}-\left(\boldsymbol{u}_{0}, \boldsymbol{w}_{\delta}\right)_{\Omega_{k}}\right\} \\
& =\sum_{k=1}^{K}\left(\boldsymbol{\lambda}, \operatorname{curl} \boldsymbol{w}_{\delta}\right) \Omega_{\Omega_{k}}-\left(\operatorname{curl} \boldsymbol{\lambda}, \boldsymbol{w}_{\delta}\right) \Omega_{\Omega_{k}} .
\end{aligned}
$$

Suppose now that $\boldsymbol{\lambda} \in H^{1}\left(\Omega_{k}\right)$ for any $k \leqslant K$. Then, after an integration by parts we get

$$
\begin{aligned}
a\left(\boldsymbol{u}, \boldsymbol{w}_{\delta}\right)-\ell\left(\boldsymbol{w}_{\delta}\right) & =\sum_{k=0}^{K} \int_{\partial \Omega_{k}}\left(\boldsymbol{w}_{\delta} \times \boldsymbol{n}\right) \cdot \boldsymbol{\lambda} \mathrm{d} \sigma \\
& =\sum_{s=0}^{m} \int_{F_{s}^{-}}\left[\boldsymbol{w}_{\delta}^{+} \times \boldsymbol{n}_{s}-\boldsymbol{w}_{\delta}^{-} \times \boldsymbol{n}_{s}\right] \cdot \boldsymbol{\lambda} \mathrm{d} \sigma \\
& =\sum_{s=0}^{m} \int_{F_{s}^{-}}\left[\boldsymbol{w}_{\delta}^{+} \times \boldsymbol{n}_{s}-\boldsymbol{w}_{\delta}^{-} \times \boldsymbol{n}_{s}\right] \cdot(\boldsymbol{\lambda}-\boldsymbol{\psi}) \mathrm{d} \sigma
\end{aligned}
$$

where $\psi$ is an arbitrary function defined on the skeleton $\mathcal{S}$ and is such that $\psi \in \mathbb{P}_{M_{s}}\left(F_{s}^{-}\right)^{3}$ for any $s \leqslant M$. It follows that for any positive and sufficiently small parameters $\rho_{k}, k=1, \ldots, K$, one has

$$
\begin{aligned}
\left|a\left(\boldsymbol{u}, \boldsymbol{w}_{\delta}\right)-\ell\left(\boldsymbol{w}_{\delta}\right)\right| & \leqslant c \sum_{s=1}^{m} \sum_{k \in \mathbb{E}(s)}\left|\int_{\partial \Omega_{k}}\left(\boldsymbol{w}_{\delta} \times \boldsymbol{n}\right) \cdot(\widetilde{\boldsymbol{\lambda}-\boldsymbol{\psi}}) \mathrm{d} \sigma\right| \\
& \leqslant c \sum_{s=1}^{m} \sum_{k \in \mathbb{E}(s)}\left\|\boldsymbol{w}_{\delta} \times \boldsymbol{n}\right\|_{H^{-1 / 2+\rho_{k}\left(\partial \Omega_{k}\right)}} \cdot\|(\widetilde{\boldsymbol{\lambda}-\boldsymbol{\psi}})\|_{H^{1 / 2-\rho_{k}\left(\partial \Omega_{k}\right)}} \\
& \leqslant c \sum_{s=1}^{m}\left(\sum_{k \in \mathbb{E}(s)} \rho_{k}^{-1 / 2}\left\|\boldsymbol{w}_{\delta} \times \boldsymbol{n}\right\|_{H^{-1 / 2+\rho_{k}}\left(\partial \Omega_{k}\right)}\|\boldsymbol{\lambda}-\boldsymbol{\psi}\|_{H^{1 / 2-\rho_{k}\left(F_{s}^{-}\right)}}\right),
\end{aligned}
$$

where $(\widetilde{\boldsymbol{\lambda}-\boldsymbol{\psi}})$ denotes the extension by zero of $\boldsymbol{\lambda}-\boldsymbol{\psi}$ to $\partial \Omega_{k}$. The term $\rho_{k}^{-1 / 2}$ is due to the norm of this extension considered as a mapping from $H^{1 / 2-\rho_{k}}\left(F_{s}^{-}\right)$into $H^{1 / 2-\rho_{k}}\left(\partial \Omega_{k}\right)$.

On the other hand, we have

$$
\left\|\boldsymbol{w}_{\delta} \times \boldsymbol{n}\right\|_{H^{-1 / 2+\rho_{k}\left(\partial \Omega_{k}\right)}} \leqslant\left\|\boldsymbol{w}_{\delta}\right\|_{H^{\rho_{k}\left(\Omega_{k}\right)}}+\left\|\operatorname{curl} \boldsymbol{w}_{\delta}\right\|_{L^{2}\left(\Omega_{k}\right)},
$$


thanks to the formula (2.1). Then

$$
\left\|\boldsymbol{w}_{\delta} \times \boldsymbol{n}\right\|_{H^{-1 / 2+\rho_{k}\left(\partial \Omega_{k}\right)}} \leqslant c N_{k}^{2 \rho_{k}}\left\|\boldsymbol{w}_{\delta}\right\|_{H\left(\operatorname{curl} ; \Omega_{k}\right)} .
$$

Finally, choosing $\rho_{k}=\left(\log N_{k}\right)^{-1}$ gives

$$
\begin{aligned}
\left|a\left(\boldsymbol{u}, \boldsymbol{w}_{\delta}\right)-\ell\left(\boldsymbol{w}_{\delta}\right)\right| & \leqslant c \sum_{s=1}^{m}\left(\sum_{k \in \mathbb{E}(s)} \rho_{k}^{-1 / 2} N_{k}^{2 \rho_{k}} M_{s}^{-\sigma_{k}-\rho_{k}}\left\|\boldsymbol{w}_{\delta}\right\|_{H\left(\operatorname{curl} ; \Omega_{k}\right)}\|\boldsymbol{\lambda}\|_{H^{\sigma_{k}+1 / 2}\left(F_{s}^{-}\right)}\right) \\
& \leqslant c \sum_{k=0}^{K}\left(\sum_{k \in \mathbb{E}(s)} \eta_{\delta}^{\sigma_{k}}\left(\log N_{k}\right)^{1 / 2} N_{k}^{-\sigma_{k}}\left\|\boldsymbol{w}_{\delta}\right\|_{H\left(\operatorname{curl} ; \Omega_{k}\right)}\|\boldsymbol{\lambda}\|_{H^{\sigma_{k}+1 / 2}\left(F_{s}^{-}\right)}\right) \\
& \leqslant c \sum_{k=0}^{K} \eta_{\delta}^{\sigma_{k}}\left(\log N_{k}\right)^{1 / 2} N_{k}^{-\sigma_{k}}\|\boldsymbol{\lambda}\|_{H^{\sigma_{k}+1}\left(\Omega_{k}\right)}\left\|\boldsymbol{w}_{\delta}\right\|_{H_{\delta}(\operatorname{curl} ; \Omega)} .
\end{aligned}
$$

3.3.3 The numerical integration error. Our task here is to give an estimate of the error due to the use of quadrature formulas for computing the integrals appearing in the discrete problem. We state the following.

Lemma 5 Suppose that the vector field $\lambda$, defined by (3.16), satisfies the assumptions of Proposition 5. Then, there exists a constant $c$ not depending either on $\delta$ or on $u$ such that

$$
\sup _{\boldsymbol{w}_{\delta} \in H_{\delta}(\operatorname{curl} ; \Omega), \boldsymbol{w} \neq 0} \frac{b\left(\boldsymbol{u}, \boldsymbol{w}_{\delta}\right)-b_{\delta}\left(\boldsymbol{u}, \boldsymbol{w}_{\delta}\right)}{\left\|\boldsymbol{w}_{\delta}\right\|_{H_{\delta}(\operatorname{curl} ; \Omega)}} \leqslant c \sum_{k=1}^{K} N_{k}^{-\sigma_{k}}\|\boldsymbol{\lambda}\|_{H^{\sigma_{k}+1}\left(\Omega_{k}\right)},
$$

where

$$
b\left(\boldsymbol{u}, \boldsymbol{w}_{\delta}\right)=a\left(\boldsymbol{u}, \boldsymbol{w}_{\delta}\right)-\ell\left(\boldsymbol{w}_{\delta}\right), \quad b_{\delta}\left(\boldsymbol{u}, \boldsymbol{w}_{\delta}\right)=a_{\delta}\left(\boldsymbol{u}, \boldsymbol{w}_{\delta}\right)-\ell_{\delta}\left(\boldsymbol{w}_{\delta}\right) .
$$

Proof. The estimate of the numerical integration is a classical exercise. In fact, consider two functions $z_{1} \in W_{\delta-1}(\Omega)^{3}, z_{2} \in W_{\delta-1}(\Omega)^{3}$. One has

$$
\begin{aligned}
\left|b\left(\boldsymbol{u}, \boldsymbol{w}_{\delta}\right)-b_{\delta}\left(\boldsymbol{u}, \boldsymbol{w}_{\delta}\right)\right| \leqslant & \mid \sum_{k=1}^{K}\left(\boldsymbol{\lambda}-z_{1}, \operatorname{curl} \boldsymbol{w}_{\delta}\right)_{\Omega_{k}}+\left(\tau \mu \boldsymbol{u}-\boldsymbol{u}_{0}+z_{2}, \boldsymbol{w}_{\delta}\right)_{\Omega_{k}} \\
& +\sum_{k=1}^{K}\left(z_{1}-\boldsymbol{\lambda}, \operatorname{curl} \boldsymbol{w}_{\delta}\right)_{\Omega_{k}, N_{k}}+\left(z_{2}-\tau \mu \boldsymbol{u}+\boldsymbol{u}_{0}, \boldsymbol{w}_{\delta}\right) \Omega_{k}, N_{k} \mid \\
\leqslant & \mid \sum_{k=1}^{K}\left(\boldsymbol{\lambda}-z_{1}, \operatorname{curl} \boldsymbol{w}_{\delta}\right)_{\Omega_{k}}+\left(z_{2}-\operatorname{curl} \boldsymbol{\lambda}, \boldsymbol{w}_{\delta}\right)_{\Omega_{k}} \\
& +\sum_{k=1}^{K}\left(z_{1}-\boldsymbol{\lambda}, \operatorname{curl} \boldsymbol{w}_{\delta}\right)_{\Omega_{k}, N_{k}}+\left(\operatorname{curl} \boldsymbol{\lambda}-z_{2}, \boldsymbol{w}_{\delta}\right)_{\Omega_{k}, N_{k}} \mid .
\end{aligned}
$$


It follows that

$$
\begin{aligned}
& \sup _{\boldsymbol{w}_{\delta} \in H_{\delta}(\operatorname{curl} ; \Omega), \boldsymbol{w}_{\delta} \neq \mathbf{0}} \frac{\left|b\left(\boldsymbol{u}, \boldsymbol{w}_{\delta}\right)-b_{\delta}\left(\boldsymbol{u}, \boldsymbol{w}_{\delta}\right)\right|}{\left\|\boldsymbol{w}_{\delta}\right\|_{H_{\delta}(\operatorname{curl} ; \Omega)}} \leqslant c\left\{\left\|\boldsymbol{\lambda}-z_{1}\right\|_{L^{2}(\Omega)}+\left\|\operatorname{curl} \boldsymbol{\lambda}-z_{2}\right\|_{L^{2}(\Omega)}\right. \\
& \left.+\left\|\mathcal{I}_{\delta} \boldsymbol{\lambda}-z_{1}\right\|_{L^{2}(\Omega)}+\left\|\mathcal{I}_{\delta}(\operatorname{curl} \boldsymbol{\lambda})-z_{2}\right\|_{L^{2}(\Omega)}\right\} \\
& \leqslant c\left\{\left\|\boldsymbol{\lambda}-z_{1}\right\|_{L^{2}(\Omega)}+\left\|\operatorname{curl} \boldsymbol{\lambda}-\boldsymbol{z}_{2}\right\|_{L^{2}(\Omega)}\right. \\
& \left.+\left\|\mathcal{I}_{\delta} \boldsymbol{\lambda}-\boldsymbol{\lambda}\right\|_{L^{2}(\Omega)}+\left\|\mathcal{I}_{\delta}(\operatorname{curl} \boldsymbol{\lambda})-\operatorname{curl} \boldsymbol{\lambda}\right\|_{L^{2}(\Omega)}\right\} .
\end{aligned}
$$

Choosing $z_{1}=\mathcal{I}_{\delta-1} \boldsymbol{\lambda}, z_{2}=\mathcal{I}_{\delta-1} \boldsymbol{\lambda}$ and using (3.7) completes the proof of (3.37).

\section{Numerical implementation}

The goal of this section is twofold. Firstly, we give some details about the numerical implementation of the system (3.13). More precisely, we prove that the matching conditions (3.8) can be written explicitly without having to resort to numerical inversion of the resulting linear system. This calculus induces the construction of an explicit basis of the discrete space $H_{\delta}(\mathbf{c u r l} ; \Omega)$.

Secondly, we present some computational results obtained with a 3D code. These results confirm the good convergence of the method and the relaxation algorithm exposed in Section 2.2.

\subsection{A basis of $H_{\delta}(\mathbf{c u r l} ; \Omega)$. The treatment of the matching conditions}

The definition of the space $H_{\delta}(\operatorname{curl} ; \Omega)$ introduced in Section 3.1 depends on the choice of the Lagrange multiplier spaces $\mathbb{M}_{\delta}^{s}\left(F_{S}\right)$. Here we consider the spaces $\mathbb{M}_{\delta}^{s}\left(F_{S}\right)$ defined by (3.9). The other choice given by (3.10) can be treated exactly by the same method.

Let $\boldsymbol{\xi}_{k}, 1 \leqslant k \leqslant G$, be the nodes of the grid $\Xi$. For each $s \in\{1, \ldots, m\}$ we set

$$
\begin{aligned}
\Lambda_{i n t} & =\left\{k \mid \boldsymbol{\xi}_{k} \notin \mathcal{S}\right\} \text { (nodes outside the skeleton), } \\
\Lambda_{s}^{+} & =\left\{k \mid \boldsymbol{\xi}_{k} \in \Xi^{\ell} \cap \mathcal{S} \text { for some } \ell \in \widetilde{\mathbb{E}}(s)\right\} \text { (nodes on the master side of the skeleton), } \\
\Lambda_{s}^{-} & =\left\{k \mid \boldsymbol{\xi}_{k} \in \Xi^{k(s)} \cap \mathcal{S}\right\} \text { (nodes on the slave side of the skeleton), } \\
\widetilde{\Lambda}_{s}^{-} & =\left\{k \in \Lambda_{s}^{-} \mid \boldsymbol{\xi}_{k} \notin \partial F_{s}^{-}\right\}, \\
\widehat{\Lambda}_{s}^{-} & =\left\{k \in \Lambda_{s}^{-} \mid \boldsymbol{\xi}_{k} \in \partial F_{s}^{-}\right\} .
\end{aligned}
$$

Notice that

$$
\operatorname{card}\left(\Lambda_{s}^{-}\right)=\left(N_{k(s)}+1\right)^{2}, \quad \operatorname{card}\left(\tilde{\Lambda}_{s}^{-}\right)=\left(N_{k(s)}-1\right)^{2}=\operatorname{dim}\left(\mathbb{P}_{N_{k(s)}-2}\right) .
$$

The grid $\Xi$ can be partitioned as follows

$$
\Xi=\left\{\boldsymbol{\xi}_{k}, k \in \Lambda_{\text {int }}\right\} \cup\left(\bigcup_{s=1}^{m}\left(\left\{\boldsymbol{\xi}_{k}, k \in \Lambda_{s}^{-}\right\} \cup\left\{\boldsymbol{\xi}_{k}, k \in \Lambda_{s}^{+}\right\}\right)\right) .
$$

Now, consider an arbitrary node $\boldsymbol{\xi}_{k}$. There exists an integer $j \leqslant K$ and a multi-index $\alpha$ such that $\boldsymbol{\xi}_{k}=\boldsymbol{\xi}_{\alpha}^{(j)}$. The basis function of $W_{\delta}(\Omega)$, associated with the node $\boldsymbol{\xi}_{k}$, is defined as

$$
\varphi_{k}(x)= \begin{cases}\ell_{\alpha}^{(j)}(\boldsymbol{x}), & \text { if } \boldsymbol{x} \in \Omega_{j}, \\ 0, & \text { elsewhere }\end{cases}
$$

where $\ell_{\alpha}^{(j)}$ is the Lagrange polynomial associated with the node $\boldsymbol{\xi}_{\alpha}^{(j)}$ in the Gauss-Lobatto grid $\Xi^{j}$. 
Observe that the jump $\left[\varphi_{k}\right]$ across the skeleton $\mathcal{S}$ vanishes if $k \in \Lambda_{\text {int }}$.

Now, any vector function $\boldsymbol{u}_{\delta} \in W_{\delta}(\Omega)^{3}$ can be written in the form

$$
\boldsymbol{u}_{\delta}=\sum_{k \in \Lambda_{\mathrm{int}}} \boldsymbol{u}_{k}^{\mathrm{int}} \varphi_{k}(\boldsymbol{x})+\sum_{s=1}^{m}\left(\sum_{k \in \Lambda_{s}^{+} \cup \Lambda_{s}^{-}}\left(u_{k}^{\mathrm{nor}} \boldsymbol{n}_{s}+\boldsymbol{u}_{k}^{\top}\right) \varphi_{k}(\boldsymbol{x})\right)
$$

where $\boldsymbol{u}_{k}^{\top} \cdot \boldsymbol{n}_{s}=0$ for any $k \in \cup_{s=1}^{m}\left(\Lambda_{s}^{+} \cup \Lambda_{s}^{-}\right)$. Moreover, $\boldsymbol{u}_{\delta}$ belongs to $H_{\delta}($ curl; $\Omega)$ if and only if its tangential components satisfy the matching conditions (3.8). These conditions can be interpreted as follows; at each face $F_{s}^{-}$of the skeleton $\mathcal{S}$, the tangential degrees of freedom $\boldsymbol{u}_{k}^{\top}, k \in \cup_{s=1}^{m}\left(\Lambda_{s}^{+} \cup \Lambda_{s}^{-}\right)$, satisfy

$$
\forall s \in\{1, \ldots, m\}, \quad \forall p \in \mathbb{P}_{N_{s}-2}\left(F_{s}^{-}\right), \quad \sum_{k \in \Lambda_{s}^{+} \cup \Lambda_{s}^{-}}\left(\int_{F_{s}^{-}}\left[\varphi_{k}\right] \cdot p(\boldsymbol{x}) \mathrm{d} \sigma\right) \boldsymbol{u}_{k}^{\top}=0 .
$$

Hence, for any $s \in\{1, \ldots, m\}$ and any $p \in \mathbb{P}_{N_{s}-2}\left(F_{s}^{-}\right)$

$$
\sum_{k \in \tilde{\Lambda}_{s}^{-}}\left(\int_{F_{s}^{-}}\left[\varphi_{k}\right] \cdot p(\boldsymbol{x}) \mathrm{d} \sigma\right) \boldsymbol{u}_{k}^{\top}=-\sum_{k \in \Lambda_{s}^{+}}\left(\int_{F_{s}^{-}}\left[\varphi_{k}\right] \cdot p(\boldsymbol{x}) \mathrm{d} \sigma\right) \boldsymbol{u}_{k}^{\top}-\sum_{k \in \widehat{\Lambda}_{s}^{-}}\left(\int_{F_{s}^{-}}\left[\varphi_{k}\right] \cdot p(\boldsymbol{x}) \mathrm{d} \sigma\right) \boldsymbol{u}_{k}^{\top} .
$$

Let $U_{s}^{+}, \widetilde{U}_{s}^{-}$and $\widehat{U}_{s}^{-}$be the vectors containing the values $\boldsymbol{u}_{k}^{\top}$ when $k$ describes $\Lambda_{s}^{+}, \tilde{\Lambda}_{s}^{-}$and $\widehat{\Lambda}_{s}^{+}$, respectively. The linear system (4.2) can be written in the form

$$
\widetilde{Q}_{s}^{-} \widetilde{U}_{s}^{-}=Q_{s}^{+} U_{s}^{+}+\widehat{Q}_{s}^{-} \widehat{U}_{s}^{-},
$$

where $\widetilde{Q}_{s}^{-}, Q_{s}^{+}$and $\widehat{Q}_{s}^{-}$are known matrices. Let $U$ be the vector containing all the values $\boldsymbol{u}_{k}^{\text {int }}$ $\left(k \in \Lambda^{\text {int }}\right), u_{k}^{\text {nor }}$ for $k \in \cup_{s=1}^{m}\left(\Lambda_{s}^{-} \cup \Lambda_{s}^{+}\right)$and $\boldsymbol{u}_{k}^{\top}$ for $k \in \cup_{s=1}^{m} \widehat{\Lambda}_{s}^{-} \cup \Lambda_{s}^{+}$. The vector field containing all the unknowns $\boldsymbol{u}_{\delta}$ at all nodes of the grid $\Xi$ is denoted by $\widetilde{U}$. Notice that $\widetilde{U}$ contains both the real unknowns already stored in $U$ and the apparent unknowns, $\boldsymbol{u}_{m}^{\top}, m \in \cup_{s=1}^{m} \widetilde{\Lambda}_{s}^{-}$. The vectors $U$ and $\widetilde{U}$ are linked by a relation of the form

$$
\widetilde{U}=Q U,
$$

where $Q$ is a rectangular matrix the rows of which are mostly those of the identity except those coming from the matrices $\left(\widetilde{Q}_{s}^{-}\right)^{-1} Q_{s}^{+}$and $\left(\widetilde{Q}_{s}^{-}\right)^{-1} \widehat{Q}_{s}^{-}$. Finally, the discrete problem (3.13) can be written in the form

$$
Q^{\top} A Q U=B
$$

where $A$ is the block matrix corresponding to the bilinear form $a_{\delta}(\cdot, \cdot)$ given by

$$
A=\left(\begin{array}{cccc}
A_{1} & 0 & 0 & 0 \\
0 & A_{2} & 0 & 0 \\
0 & 0 & \ddots & 0 \\
0 & 0 & 0 & A_{K}
\end{array}\right) .
$$

Here $A_{k}, k=1, \ldots, K$, is the stiffness matrix associated with the local bilinear form

$$
a_{k}(\boldsymbol{u}, \boldsymbol{v})=\left(\epsilon^{-1} \operatorname{curl} \boldsymbol{u}, \operatorname{curl} \boldsymbol{v}\right)_{\Omega_{k}, N_{k}}+\tau(\mu \boldsymbol{u}, \boldsymbol{v})_{\Omega_{k}, N_{k}} .
$$


Since the final matrix $\widehat{A}=Q^{\top} A Q$ is positive definite and symmetric, one can use a usual iterative method, e.g. Conjugate Gradient, for solving the linear system (4.4).

Let us prove now that the matching linear systems (4.3) can be inverted manually without resort to numerical inversion. We provide by this way a basis of the space $H_{\delta}(\mathbf{c u r l} ; \Omega)$. We suppose for simplicity that $\left.F_{s}^{-}=\right]-1,1\left[^{2} \times\{0\}\right.$. We can write

$$
\boldsymbol{u}_{\delta}=\sum_{k \in \Lambda_{\mathrm{int}}} \boldsymbol{u}_{k}^{\mathrm{int}} \varphi_{k}+\sum_{s=1}^{m}\left(\sum_{k \in \Lambda_{s}^{+} \cup \Lambda_{s}^{-}} u_{k}^{\mathrm{nor}} \varphi_{k} \boldsymbol{n}_{s}+\sum_{k \in \Lambda_{s}^{+} \cup \widehat{\Lambda}_{s}^{-}} \boldsymbol{u}_{k}^{\top} \widetilde{\varphi}_{k}\right) .
$$

Here the basis functions $\widetilde{\varphi}_{k}$ are defined by

$$
\widetilde{\varphi}_{k}=\varphi_{k}-\sum_{j \in \widetilde{\Lambda}_{s}^{-}} q_{k, j} \varphi_{j}, \quad 1 \leqslant s \leqslant m, k \in \Lambda_{s}^{+} \cup \widehat{\Lambda}_{s}^{-},
$$

where the coefficients $q_{k, j}$ are chosen so that

$$
\int_{F_{s}^{-}}\left[\widetilde{\varphi}_{k}\right] \cdot q \mathrm{~d} \sigma=0, \quad \forall q \in \mathbb{P}_{N_{s}-2}\left(F_{s}^{-}\right) .
$$

It is worth noting that for any $s \leqslant m$ and $k \in \Lambda_{s}^{+}$we have

$$
\tilde{\varphi}_{k}= \begin{cases}\varphi_{k}, & \text { on the master side of } F_{s}^{-}, \\ -\sum_{j \in \tilde{\Lambda}_{s}^{-}} q_{k, j} \varphi_{j}, & \text { on the slave side of } F_{s}^{-} .\end{cases}
$$

It follows that the vector $V_{k}=\left(q_{k, 1}, \ldots, q_{k, \theta}\right), \theta=\operatorname{card}\left(\widetilde{\Lambda}_{s}^{-}\right)$, is a solution of a linear system of the form $\widetilde{Q} V_{k}=B_{k}$.

For any $k \in \Lambda_{s}^{+} \cup \widehat{\Lambda}_{s}^{-}$, define on $F_{s}^{-}$the functions

$$
\psi_{k}=\sum_{j \in \widetilde{\Lambda}_{s}^{-}} q_{k, j} \varphi_{j}^{-}, \quad \tilde{\psi}_{k}= \begin{cases}\varphi_{k}^{-}, & \text {if } k \in \widehat{\Lambda}_{s}^{-}, \\ \pi_{N_{s}} \varphi_{k}^{+}, & \text {if } k \in \Lambda_{k}^{+},\end{cases}
$$

where $\varphi_{k}^{+}$(resp. $\varphi_{k}^{-}$) denotes the value of $\varphi_{k}$ on the master (resp. the slave) side of $F_{s}^{-}$and $\pi_{N_{s}}$ is the orthogonal projector from $L^{2}\left(F_{s}^{-}\right)$onto $\mathbb{P}_{N_{s}}\left(F_{s}^{-}\right)$. Notice that for each $k \in \Lambda_{s}^{+}$one has

$$
\tilde{\psi}_{k}=\sum_{\ell, j=1}^{N_{s}} \frac{(2 \ell+1)(2 j+1)}{4}\left(\int_{F_{s}^{-}} \varphi_{k}^{+}(x, y) \cdot L_{\ell}(x) L_{j}(y) \mathrm{d} x \mathrm{~d} y\right) L_{\ell}(x) L_{j}(y) \mathrm{d} x \mathrm{~d} y .
$$

The matching condition (4.6) can be rewritten in the form

$$
\psi_{k}-\tilde{\psi}_{k} \in \mathbb{P}_{N_{s}-2}^{\perp}\left(F_{s}^{-}\right),
$$

where $\mathbb{P}_{N_{s}-2}^{\perp}\left(F_{s}^{-}\right)$is the orthogonal projector of $\mathbb{P}_{N_{s}-2}\left(F_{s}^{-}\right)$in $\mathbb{P}_{N_{s}}\left(F_{s}^{-}\right)$. Since

$$
\begin{aligned}
\mathbb{P}_{N_{s}-2}^{\perp}\left(F_{s}^{-}\right)= & \left\{p(x) L_{j}(y)+q(y) L_{k}(x), p \in \mathbb{P}_{N_{s}}(]-1,1[x), q \in \mathbb{P}_{N_{s}}(]-1,1[y),\right. \\
& \left.N_{s}-1 \leqslant j, k \leqslant N_{s}\right\},
\end{aligned}
$$


one gets easily to the formula

$$
\begin{aligned}
\psi_{k}= & \tilde{\psi}_{k}-\frac{1}{2} \tilde{\psi}_{k}(x, 1)\left(L_{N_{s}}(y)+L_{N_{s}-1}(y)\right)-\frac{(-1)^{N_{s}}}{2} \tilde{\psi}_{k}(x,-1)\left(L_{N_{s}}(y)-L_{N_{s}-1}(y)\right) \\
& -\frac{1}{2} \tilde{\psi}_{k}(1, y)\left(L_{N_{s}}(x)+L_{N_{s}-1}(x)\right)-\frac{(-1)^{N_{s}}}{2} \tilde{\psi}_{k}(-1, y)\left(L_{N_{s}}(x)-L_{N_{s}-1}(x)\right) \\
& -\left(\frac{\delta}{2} L_{N_{s}-1}(x)+\frac{\beta}{2} L_{N_{s}}(x)\right) L_{N_{s}-1}(y)-\left(\frac{\gamma}{2} L_{N_{s}-1}(x)+\frac{\lambda}{2} L_{N_{s}}(x)\right) L_{N_{s}-1}(y),
\end{aligned}
$$

where

$$
\begin{aligned}
2 \lambda & =\tilde{\psi}_{k}(1,1)+(-1)^{N_{s}}\left(\tilde{\psi}_{k}(-1,1)+\tilde{\psi}_{k}(1,-1)\right)+\tilde{\psi}_{k}(-1,-1), \\
2 \beta & =\tilde{\psi}_{k}(1,1)+(-1)^{N_{s}}\left(\tilde{\psi}_{k}(-1,1)-\tilde{\psi}_{k}(1,-1)\right)-\tilde{\psi}_{k}(-1,-1), \\
2 \gamma & =\tilde{\psi}_{k}(1,1)+(-1)^{N_{s}}\left(\tilde{\psi}_{k}(-1,1)+\tilde{\psi}_{k}(1,-1)\right)-\tilde{\psi}_{k}(-1,-1), \\
2 \delta & =\tilde{\psi}_{k}(1,1)+(-1)^{N_{s}}\left(\tilde{\psi}_{k}(-1,1)-\tilde{\psi}_{k}(1,-1)\right)+\tilde{\psi}_{k}(-1,-1) .
\end{aligned}
$$

Finally, observe that

$$
\left.\forall k \in \Lambda_{s}^{+} \cup \widehat{\Lambda}_{s}^{-}, \quad \forall j \in \widetilde{\Lambda}_{s}^{-}, \quad q_{k, j}=\psi_{i} \quad \begin{array}{c}
k(s) \\
\alpha(j)
\end{array}\right),
$$

where $\boldsymbol{\xi}_{\alpha(j)}^{k(s)} \in F_{s}^{-}$is the node associated with the function $\varphi_{j}$. This $\quad$ is the inversion of (4.3) and the construction of a basis of $H_{\delta}(\operatorname{curl} ; \Omega)$.

\subsection{Computational tests}

Our purpose here is to show some numerical experiments obtained with a 3D code for solving the discrete system (3.13). The aim of these tests is to compare the computational results with the numerical analysis given above. The domain of computation is the cube $\Omega=]-1,1\left[^{3}\right.$ decomposed into three sub-domains

$$
\left.\Omega_{1}=\right]-1,1[2 \times]-1,0\left[, \quad \Omega_{2}=\right]-1,0[\times]-1,1[\times] 0,1\left[, \quad \Omega_{3}=\right] 0,1[\times]-1,1[\times] 0,1[.
$$

This decomposition is clearly non-conforming. The mortar faces are

$$
\begin{array}{ll}
\left.F_{1}=\right]-1,0[\times]-1,1[\times\{0\}, & k(1)=2, \\
\left.F_{2}=\right] 0,1[\times]-1,1[\times\{0\}, & k(2)=3, \\
\left.F_{3}=\{0\} \times\right]-1,1[\times]-1,0[, & k(3)=3 .
\end{array}
$$

Figure 3 shows a vertical section of the domain $\Omega=\Omega_{1} \cup \Omega_{2} \cup \Omega_{3}$.

The tests we make here are gathered into two parts. The first tests concern the approximation of the problem (3.11) by the mortar spectral method exposed above (Examples 1 and 2). The second tests are devoted to approximating the harmonic problem (2.5) by means of the relaxation scheme exposed in Section 2.2. The mortar method is used at each iteration of that scheme. 


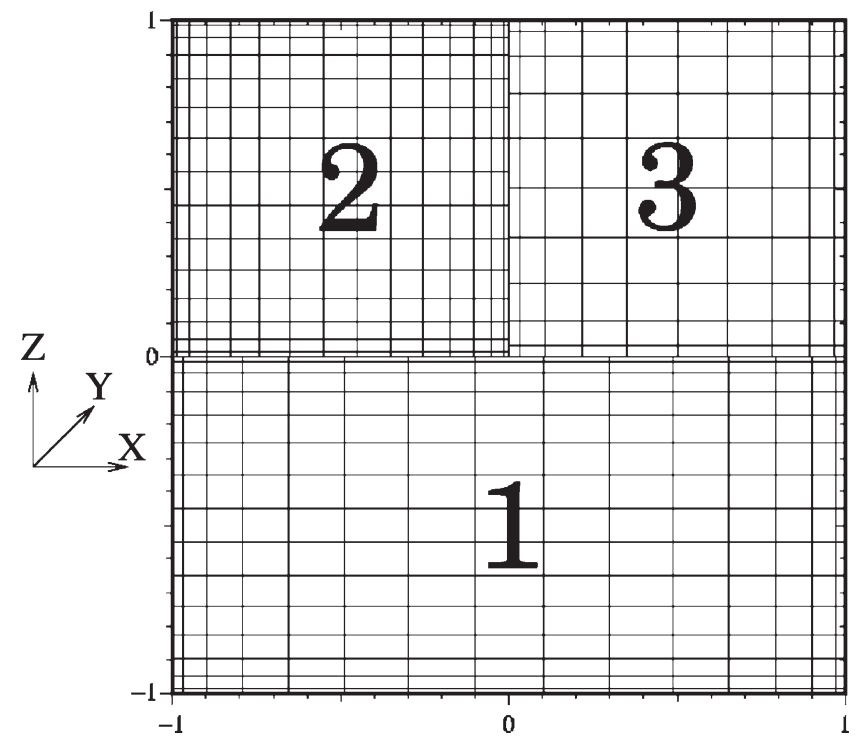

FIG. 3. A vertical section of the 3D domain used for the numerical tests with a 15-15-10 Gauss-Lobatto grid.

4.2.1 Tests on the convergence of the mortar method. In all the examples here, the parameters $\epsilon$ and $\mu$ are piecewise constant, namely

$$
\mu(\boldsymbol{x})=\mu_{k}, \quad \epsilon(\boldsymbol{x})=\epsilon_{k} \text { in } \Omega_{k}, \quad k=1,2,3 .
$$

For simplicity we say that a discretization is $m-n-k$, where $m, n, k$ are three integers if $N_{1}=m$, $N_{2}=n$ and $N_{3}=k$.

EXAmple 1: The Homogeneous CASE The analytical example we use is given by

$$
\boldsymbol{u}=\mu^{-1} v, \quad j=\mu^{-1} \operatorname{curl} v+\tau \epsilon w,
$$

where

$$
\boldsymbol{v}(x, y, z)=\left(\begin{array}{l}
f(x) f^{\prime}(y) f^{\prime}(z) \\
4 f^{\prime}(x) f(y) f^{\prime}(z) \\
-5 f^{\prime}(x) f^{\prime}(y) f(z)
\end{array}\right), \quad \boldsymbol{w}=\left(\begin{array}{l}
3 f^{\prime}(x) f(y) f(z) \\
-2 f(x) f^{\prime}(y) f(z) \\
-f(x) f(y) f^{\prime}(z)
\end{array}\right)
$$

and $f(t)=(\sinh 2) \sinh t-(\sinh 1) \sinh (2 t)$. Here we choose $\epsilon_{k}=\mu_{k}=1$ for $k \in\{1,2,3\}$.

In Fig. 4 the three components of the vector field $\boldsymbol{u}$ at the section $y=1 / 2$ are displayed. The same components are shown in the same figure after a computation by the mortar method with a 10-8-8 grid. These figures show, on the one hand, the continuity of the tangential trace across the mortars which is ensured by the matching conditions (3.8). On the other hand, they display the continuity of the normal trace which is implicit to the original equation.

Figure 5 shows the space distribution of the error when a 10-8-6 discretization is used. One can observe that the interface between sub-domains 1 and 2 is quasi-transparent since the error in its neighbourhood is very small. However, the error at interfaces between the sub-domain 3 and the sub-domains 1 and 2 is more significant. This is not due to the mortar matching conditions but to the diffusion of errors coming from sub-domain 3 where the grid is rough. 

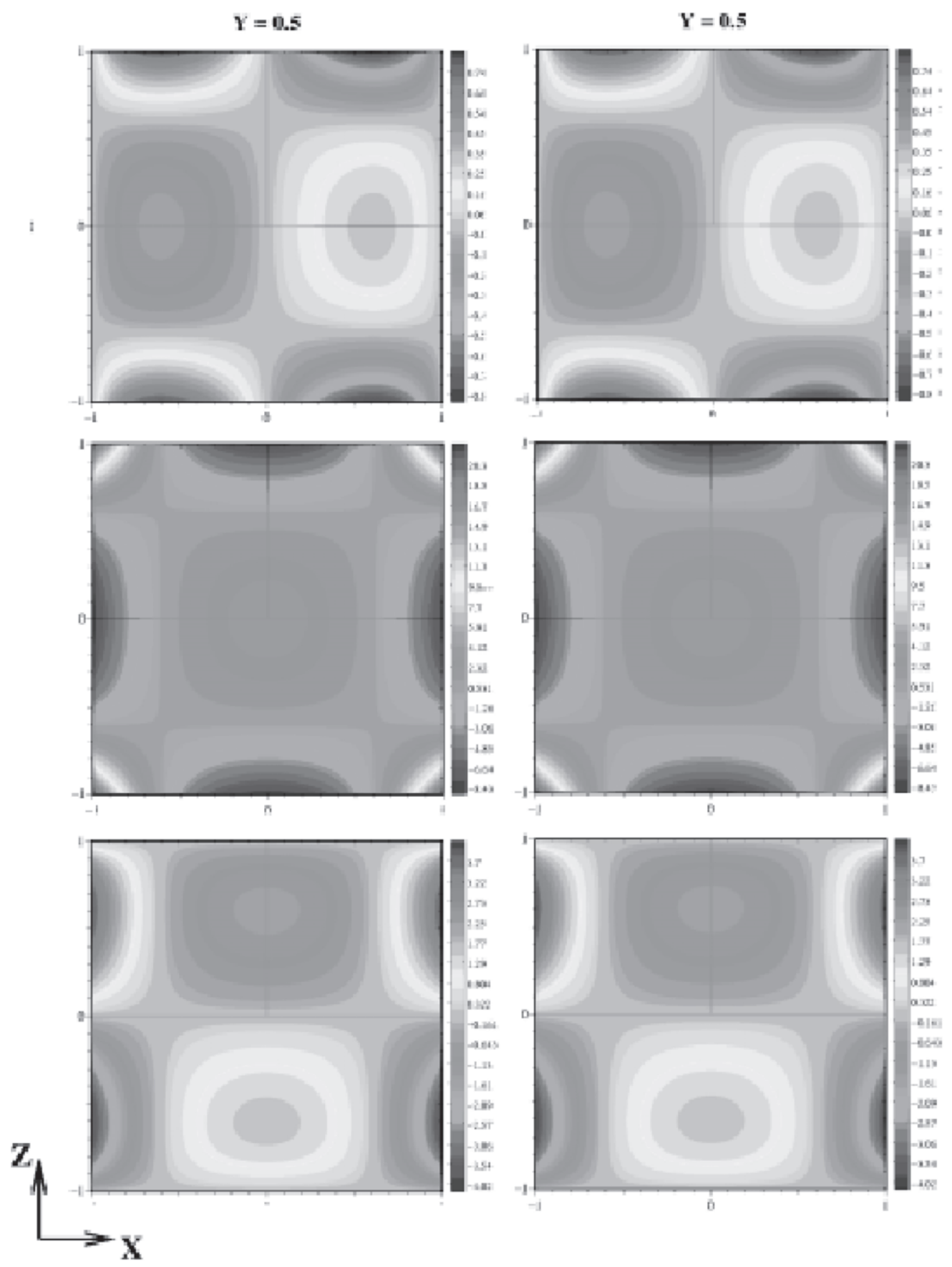

FIG. 4. Example 1. The three components of the exact solution at the section $y=1 / 2$ (left) and of the approximate one (right) at the section $y=1 / 2$. The computation of the approximate solution is done with a $10-8-8$ mortar method. 

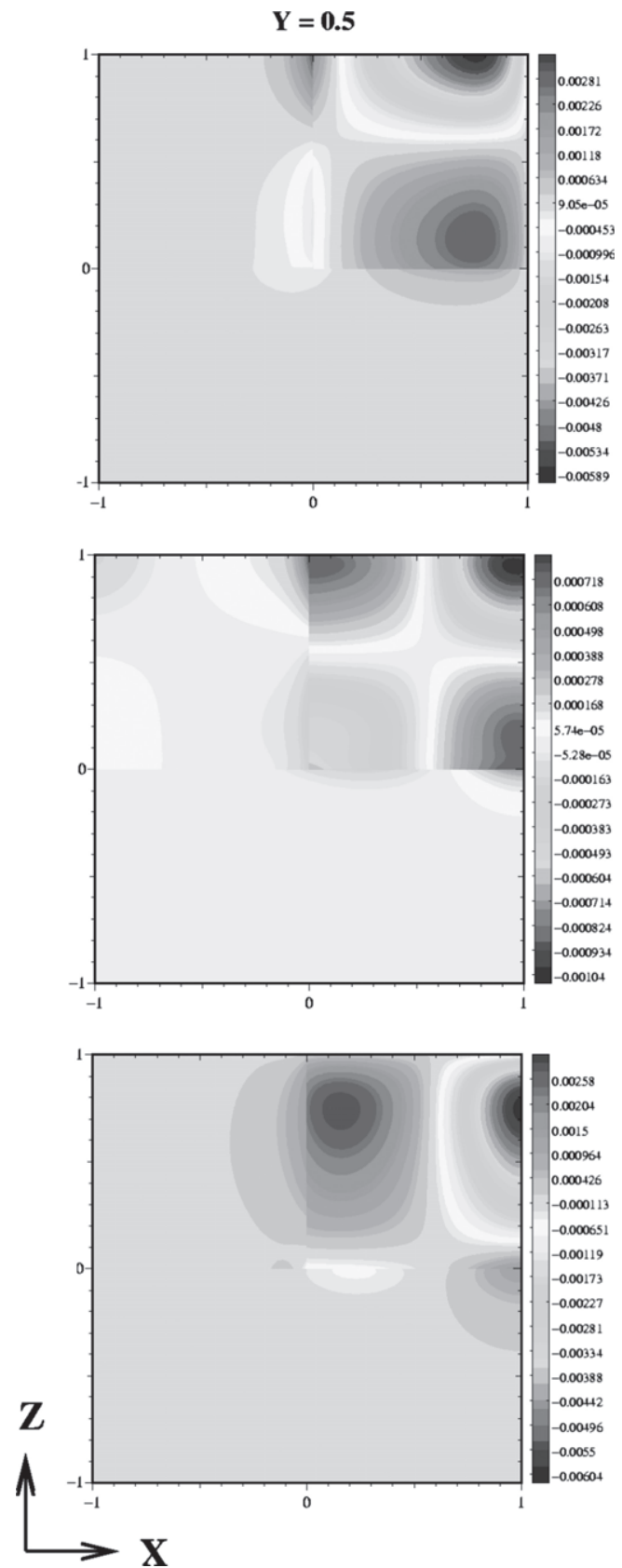

FIG. 5. Example 1. The distribution at $y=1 / 2$ of the numerical error when a $10-8-6$ mortar method is used for computing the approximate solution. 

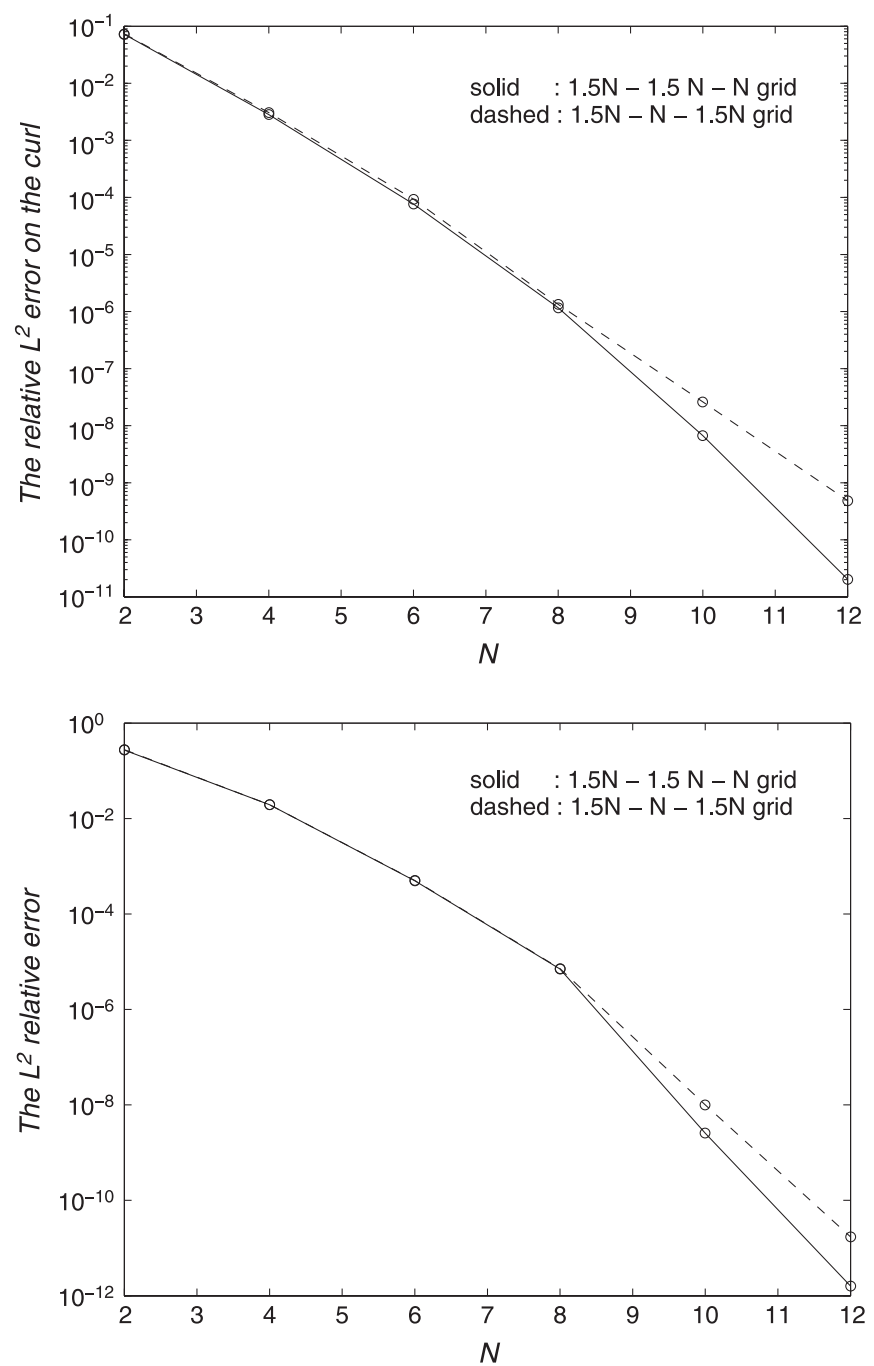

FIG. 6. Example 1. The relative $L^{2}$ error on the curl (in a logarithmic scale) versus $N$ for a $3 N / 2-3 N / 2-N$ discretization (solid line) and a $3 N / 2-N-3 N / 2$ discretization (dashed line) (left). The relative $L^{2}$ error (in a logarithmic scale) versus $N$ for the same discretizations (right).

In Figs 6 and 7 the relative $L^{2}$ error on $\operatorname{curl} \boldsymbol{u}$, on $\boldsymbol{u}$ and the $L^{2}$ norm of $\operatorname{div} \boldsymbol{u}$ (the latter is divided by the $L^{2}$ norm of $\operatorname{curl} \boldsymbol{u}$ ) are displayed, respectively, versus a parameter $N$ for $1.5 N-1.5 N-N$ and $1.5 N-N-1.5 N$ discretizations.

Example 2: The Heterogeneous Case In this second example, we keep the same exact solution as in Example 1, but we use heterogeneous physical parameters $\epsilon$ and $\mu$. Namely, we take

$$
\begin{aligned}
& \epsilon_{1}=1.0, \quad \epsilon_{2}=\epsilon_{3}=1.5, \\
& \mu_{1}=1.0, \quad \mu_{2}=2.0, \quad \mu_{3}=0.5 .
\end{aligned}
$$




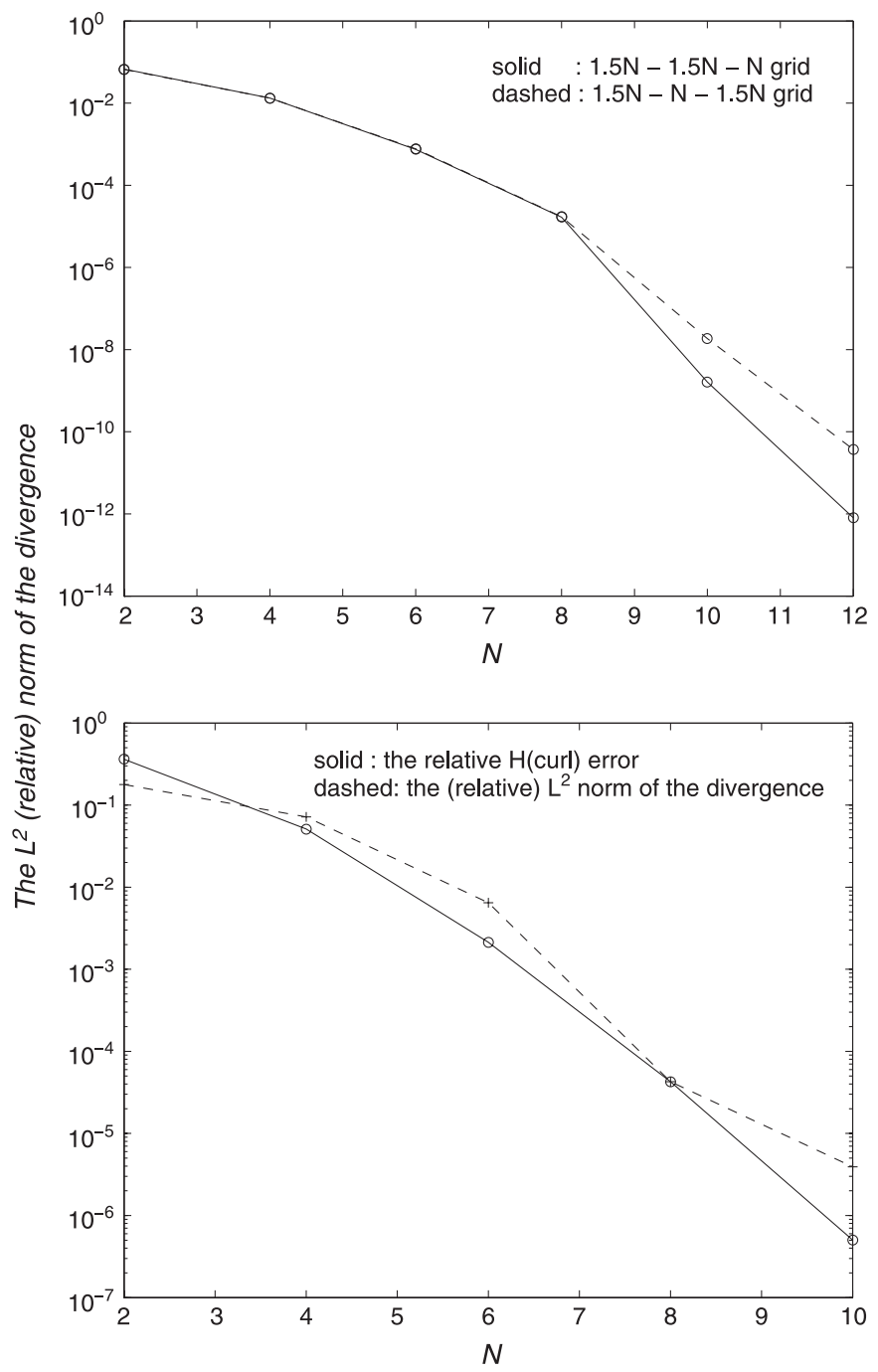

FIG. 7. Example 1. The relative $L^{2}$ norm of the divergence (in a logarithmic scale) versus $N$ for a $3 N / 2-3 N / 2-N$ discretization (solid line) and a $3 N / 2-N-3 N / 2$ discretization (dashed line) (left). Example 2. The relative $H$ (curl) norm of the error (solid line) and the $L^{2}$ norm of the divergence (dashed line) (in a logarithmic scale) versus $N$ for a $3 N / 2-3 N / 2-N$ discretization (right).

The three components of this heterogeneous solution are displayed in Fig. 8 (on the left) at the section $y=1 / 2$. The distribution of the error in each component is also displayed at the same section (on the right).

Figure 7 shows the behaviour of the $H$ (curl) norm of the error and the $L^{2}$ norm of the divergence versus $N$ when a $1.5 N-1.5 N-N$ discretization is used. The decay of these errors confirms the convergence of the method in the case of heterogenous media, provided that the solution is sufficiently smooth.

4.2.2 Tests on the convergence of the iterative scheme for a harmonic system. Our task here is to confirm numerically the convergence of the iterative procedure proposed in Section 2.2 for solving the 

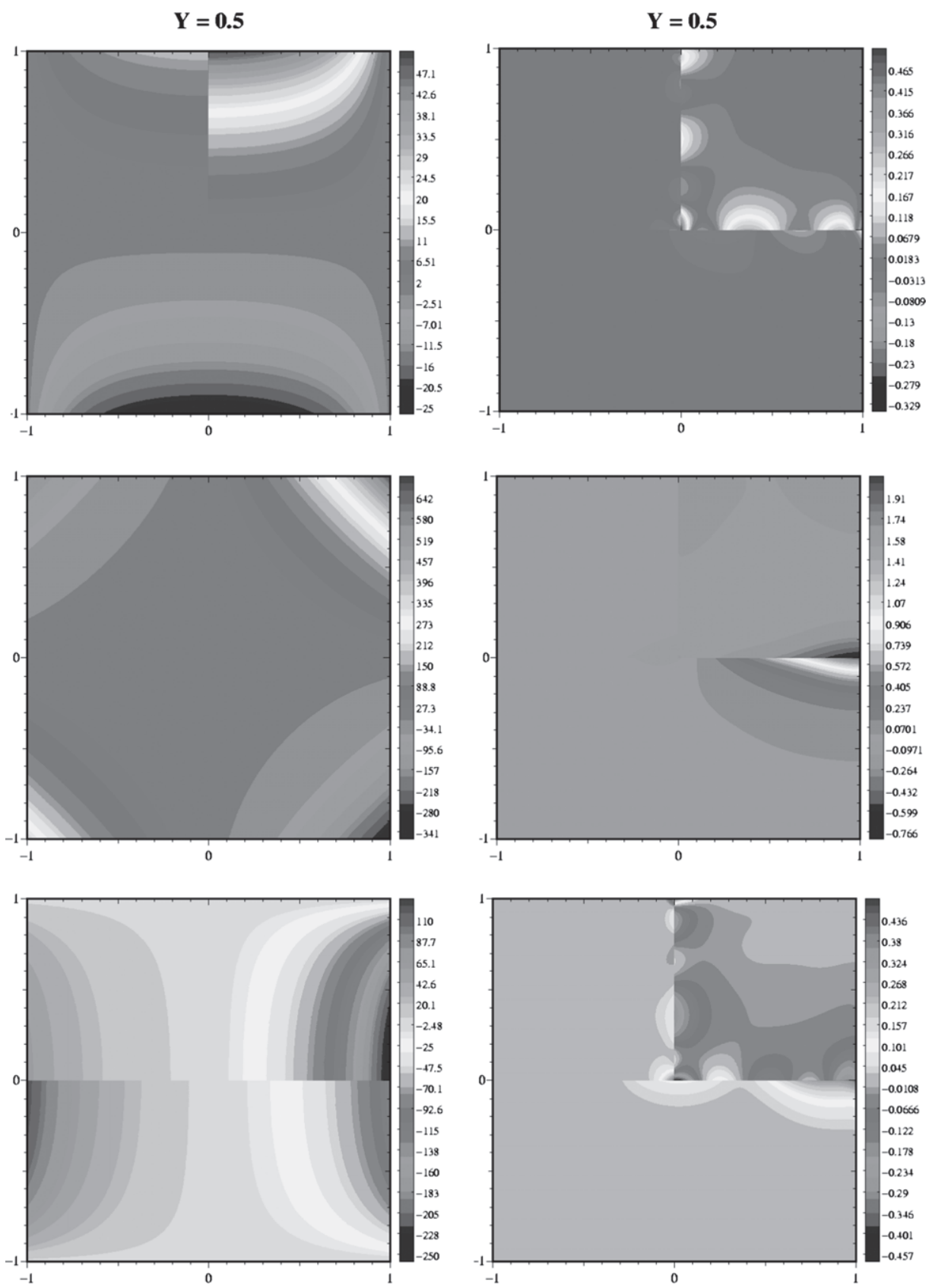

FIG. 8. Example 2. The three components of the exact heterogeneous solution (left). The distribution of the numerical error when a 9-5-6 mortar spectral method is used (right). 

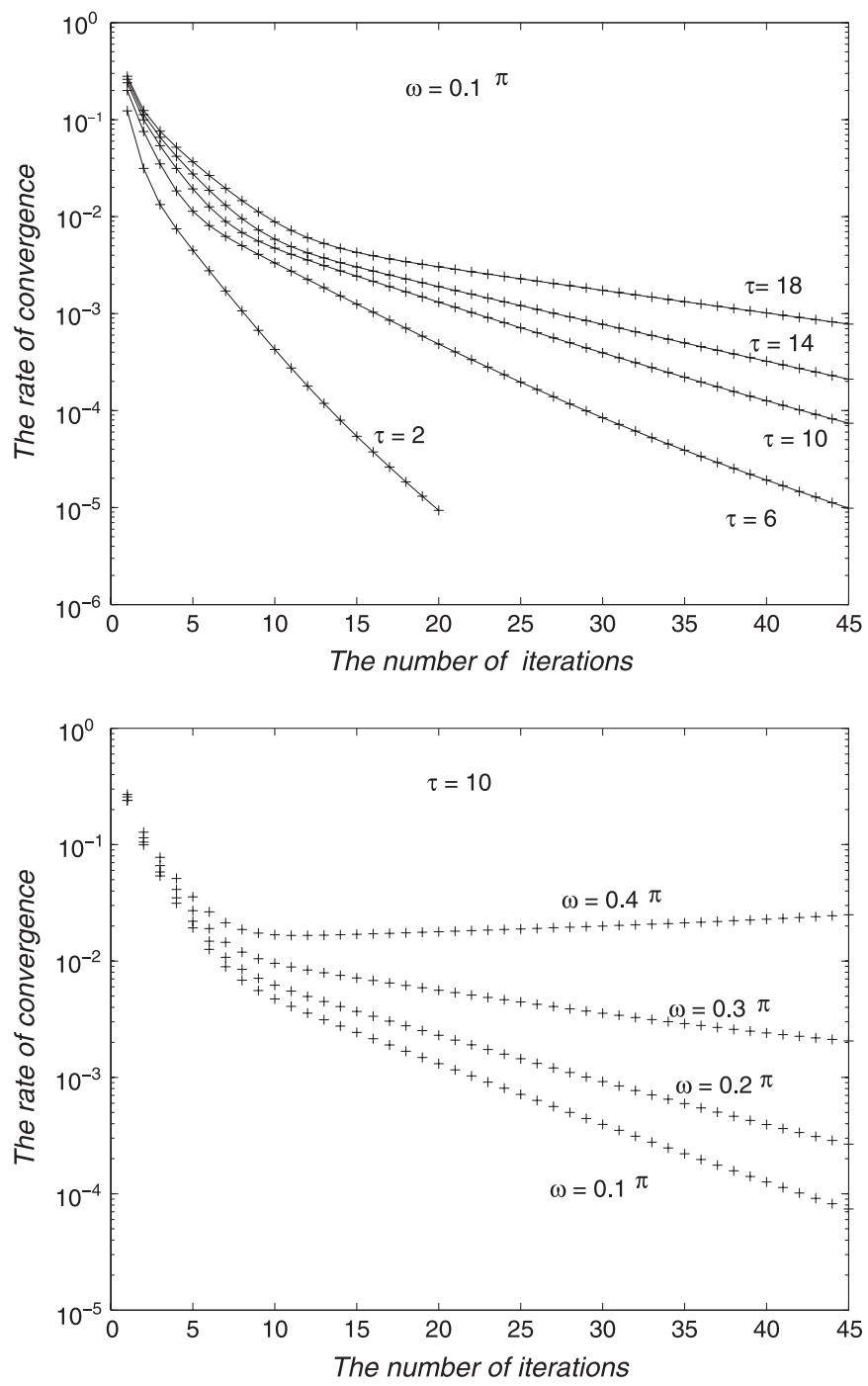

FIG. 9. The rate of convergence $\left\|\boldsymbol{u}_{n+1}-\boldsymbol{u}_{n}\right\|_{X_{T}(\mu ; \Omega)} /\left\|\boldsymbol{u}_{n}\right\|_{X_{T}(\mu ; \Omega)}$ versus the number of iterations for several values of the parameter $\tau$ ( $\omega$ is fixed: $\omega=0.1 \pi$ ) (left). The rate of convergence versus the number of iterations for several values of $\omega(\tau$ is fixed: $\tau=10$ ) (right). A 7-5-5 discretization is used for all the tests.

harmonic problem (2.5). In all the tests, the parameters $\epsilon$ and $\mu$ are supposed constant. The current density $\boldsymbol{j}$ is given by

$$
j=\mu^{-1} \operatorname{curl} v-\omega^{2} \epsilon w
$$

where $\boldsymbol{v}$ and $\boldsymbol{w}$ are given by (4.9). The corresponding solution of (2.5) is $\boldsymbol{u}=\mu^{-1} \boldsymbol{v}$.

The discretization parameters are also fixed; $N_{1}=7, N_{2}=5, N_{3}=5$. Figure 9 shows the rate of convergence

$$
\frac{\left\|\boldsymbol{u}_{n+1}-\boldsymbol{u}_{n}\right\|_{X_{T}(\mu ; \Omega)}}{\left\|\boldsymbol{u}_{n}\right\|_{X_{T}(\mu ; \Omega)}}
$$


versus the iteration number $n$ for several values of $\tau$ (with $\omega=0.1 \pi$ ) and for several values of $\omega$ $(\tau=10)$. It is quite clear that the smaller $\tau$ and $\omega$ are, the faster is the convergence. Furthermore, there exists a value $\omega_{\delta} \approx 0.4 \pi$ such that the algorithm does not converge if $\omega>\omega_{\delta}$. All these observations confirm the predictions of Proposition 1. It is worth noting that the error does not vary significantly with the parameter $\tau$. However, this parameter has a significant influence on the spectral properties of the matrix $A, \hat{A}=Q^{\top} A Q$. More precisely, for small (but positive) values of $\tau$, the convergence of the CG algorithm used for inverting the linear system becomes slow. Nevertheless, in such a situation one can use preconditioners to get rapid convergence.

\section{REFERENCES}

Beck, R., Deuflhard, P., Hiptmair, R., Hoppe, R. \& Wohlmuth, B.(1999) Adaptative multilevel methods for edge element discretization of Maxwell's equations. Surveys Math. Indust., 8, 271-312.

BeCK, R. \& Hiptmair, R. (1999) Multilevel solution of the time-harmonic Maxwell's equations based on edge elements. Int. J. Numer. Meth. Engng., 45, 901-920.

Belhachmi, Z. \& Bernardi, C. (1994) Resolution of fourth order problems by the mortar element method. Comput. Meth. Appl. Mech. Engng., 116, 53-58.

Ben Belgacem, F., Buffa, A. \& MAdAY, Y. (2001) The mortar finite element method for 3D Maxwell equations: first results. SIAM J. Numer. Anal., 39, 880-901.

Berger, A., Scott, R. \& Strang, G. (1972) Approximate boundary conditions in the finite element method. Symp. Math., 10, 295-313.

Bernardi, C., DÉBit, N. \& MAdAy, Y. (1990a) Coupling finite element and spectral methods; first results. Math. Comp., 54, 21-39.

Bernardi, C. \& MAdAy, Y. (1992) Approximation Spectrale de Problèmes aux Limites Elliptiques. Collection Mathématiques et Applications. Paris: Springer.

Bernardi, C. \& Maday, Y. (1994) Basic Results on Spectral Methods. Laboratoire d'Analyse Numérique, Université Paris VI. Preprint R94022.

Bernardi, C., Maday, Y. \& Patera, A. T. (1994) A new nonconforming approach to domain decomposition: the mortar element method. Nonlinear Partial Differential Equations and Their Applications. Collège de France seminar. Vol. XI (Paris, 1989-1991), 13-51, Pitman Res. Notes Math. Ser., 299, Longman Sci. Tech., Harlow.

Bertoluzza, S. \& Perrier, V. (2001) The mortar method in the wavelet context. ESAIM: M2AN, 35, 647-673.

Boulmezaoud, T. Z. \& El RhABI, M. (2003) On time-harmonic Maxwell's equations in Lipschitz and multiplyconnected domains of $\mathbb{R}^{3}$. Monogr. Semin. Mat. García Galdeano, 27, 127-134.

Buffa, A., Hiptmair, R., von Petersdorff, T. \& Schwab, C. (2003) Boundary elements methods for Maxwell transmission problems in Lipstchitz domains. Numer. Math., 95, 459-485.

CANUto, C. \& QUARTERONi, A. (1982) Approximation results for orthogonal polynomials in Sobolev spaces. Math. Comput., 38, 67-86.

Costabel, M. (1991) A coercive bilinear form for Maxwell's equations. J. Math. Anal. Appl., 157, 527-541.

Costabel, M. \& Dauge, M. (1999) Singularities of Maxwell interface problems. Modél. Math. Anal. Numér., 33, 627-649.

Costabel, M. \& Dauge, M. (2000) Singularities of electromagnetic fields in polyhedral domains. Arch. Ration. Mech. Anal., 151, 221-276.

Dautray, R. \& LiOns, J.-L. (1988) Analyse Mathématique et Calcul numérique, tome 5. Paris: Masson.

DAVIS, P. J. \& RABINOWITZ, P. (1985) Methods of Numerical Integration. Orlando: Academic Press.

DÉBIT, N. \& MADAY, Y. (1987) The coupling of spectral and finite element methods for the approximation of the Stokes problem, in proceedings of the 8th Joint France-Italy-U.S.S.R. Symposium of Computational Mathematics and Applications. Pavia. 
EL Rhabi, M. (2002) Analyse numrique et discrtisation par lments spectraux avec joints des quations tridimensionnelles de l'lectromagntisme. Ph.D. Thesis, Université Pierre et Marie Curie, Paris.

Fisher, P. F. \& Patera, A. T. (1991) Parallel spectral element solution of the Stokes problem. J. Comput. Phys., 92, 380-421.

HiptmaiR, R. (1999) Multigrid method for Maxwell's equations. SIAM J. Numer. Anal., 36, 204-225.

Hoppe, R. H. W. (1981) A penalty method for approximate solution of stationary Maxwell equations. Numer. Math., 36, 389-403.

Hoppe, R. H. W. (1982) Crank-Nicolsen-Galerkin approximation for Maxwell's equations. Math. Meth. Appl. Sci., 4, 123-130.

LEIS, R. (1986) Initial Boundary Value Problems in Mathematical Physics. B. G. Teubner, Stuttgart.

Monk, P. (1992a) A finite element method for approximating the time-harmonic Maxwell's equations. Numer. Math., 63, 243-261.

Monk, P. (1992b) Analysis of a Finite Element Method for Maxwell's Equations. SIAM J. Numer. Anal., 29, 714-729.

Monk, P. (1993) An analysis of Nédélec method for the spatial discretization of Maxwell's equations. J. Comput. Appl. Math., 47, 101-121.

Monk, P. (2003) Finite Element Methods for Maxwell's Equations. New York: Oxford University Press.

RAPETTI, F. (2000) Approximation des équations de la magnétodynamique en domaine tournant par la méthode des éléments avec joints. Ph.D. Thesis, Université Paris VI.

SzEGö, G. (1978) Orthogonal polynomials. Providence, RI: Colloquium Publications.

Toselli, A. (2000) Overlapping Schwarz methods for Maxwell's equations in three dimensions. Numer. Math., 86, 733-752.

Toselli, A., Widlund, O. B. \& Wohlmuth, B. (2001) An iterative substructuring method for Maxwell's equations in two dimensions. Math. Comp., 70, 935-949.

Wohlmuth, B. I. (2000) A mortar finite element method using dual spaces for the lagrange multiplier. SIAM J. Numer. Anal., 38, 989-1012. 